\title{
Disconnect between sputum neutrophils and other measures of airway inflammation in asthma
}

\section{To the Editor:}

Asthma heterogeneity has been described by the nature and intensity of granulocytic infiltration into the airways [1, 2]. Sputum, endobronchial biopsies and bronchoalveolar lavage (BAL) sample different anatomical regions of the airways. Few studies have directly compared the inflammatory cell infiltrates in these regions within a large cohort of moderate-severe asthma patients using standard techniques.

In the BOBCAT (Bronchoscopic exploratory research study Of Biomarkers in Corticosteroid-refractory AsThma) study, we sampled multiple airway compartments concurrently, enabling us to evaluate relationships between granulocytic infiltrates within and between compartments in a large cohort of moderate-severe adult asthma patients. This prospective multicentre observational study was conducted in four visits over a 4-6 week period, as described previously [3].

The patients included had moderate-severe persistent asthma (forced expiratory volume in $1 \mathrm{~s}$ (FEV1) 40-80\% predicted and Asthma Control Questionnaire (ACQ) [4] score $>1.5$ ) and, within the past 5 years, evidence of $>12 \%$ post-bronchodilator reversibility or a provocative concentration of methacholine causing a $20 \%$ decline in FEV $1 \leqslant 8 \mathrm{mg} \cdot \mathrm{mL}^{-1}$ despite high-dose inhaled corticosteroid (ICS) $\left(\geqslant 1000 \mu \mathrm{g} \cdot \mathrm{day}^{-1}\right.$ fluticasone propionate equivalent) with or without long-acting $\beta_{2}$-adrenergic agonist therapy. Key exclusion criteria included initiation or increase in systemic steroid use 30 days prior to screening, chronic or recent (within the past 30 days) use of immunosuppressive therapies, or other active lung disease. Patients had a prior established diagnosis of moderate-severe asthma for $\geqslant 6$ months prior to screening while receiving a stable dose regimen ( $>6$ weeks) of a high-dose ICS. Allowed concomitant medications included leukotriene receptor antagonists and oral corticosteroids.

78 patients with confirmed moderate-severe asthma were enrolled at 18 sites; 67 (86\%) completed the study. All patients had persistently impaired lung function and uncontrolled symptoms despite high-dose ICS treatment of $\geqslant 1000 \mu \mathrm{g} \cdot \mathrm{day}^{-1}$ fluticasone propionate equivalent. 11 (14\%) patients did not complete the study, due to an adverse event $(n=1)$, physician's decision $(n=5)$, subject's decision $(n=3)$ or the sponsor's decision $(n=2)$. One patient experienced increased bronchospasm following bronchoscopy, requiring additional observation and oral corticosteroid treatment prior to being discharged home from the final study visit.

We enumerated sputum, tissue and BAL eosinophils and neutrophils using standard techniques as described previously $[5,6]$. Both granulocyte types spanned broad ranges and had a unimodal distribution in each compartment (fig. 1). The majority of nonsquamous cells in sputum from most subjects were neutrophils, while a much smaller percentage of sputum cells were eosinophils. The majority of BAL cells were macrophages (data not shown), with eosinophils and neutrophils comprising $<10 \%$ of total BAL cells in most cases.

Within each compartment, eosinophils and neutrophils were significantly intercorrelated. However, these correlations were positive in biopsy tissue and BAL but negative in sputum (fig. 1). The correlation within any single compartment was greatest in biopsy tissue (Spearman's rank correlation $(\mathrm{rs})=0.68, \mathrm{p}<0.0001$ ). Across compartments, sputum eosinophils were significantly, albeit weakly, positively correlated with both biopsy tissue eosinophils ( $\mathrm{rs}=0.36, \mathrm{p}<0.05$ ) and BAL eosinophils ( $\mathrm{rs}=0.33, \mathrm{p}<0.05$ ), while neutrophils were not intercorrelated across any compartments. Sputum neutrophils were negatively correlated with tissue eosinophils $(\mathrm{rs}=-0.37, \mathrm{p}<0.01)$ and BAL eosinophils $(\mathrm{rs}=-0.34, \mathrm{p}<0.05)$. These findings show generally positive relationships between eosinophils and neutrophils within and across airway compartments, with the exception of sputum neutrophil percentage.

Serum periostin and exhaled nitric oxide fraction ( $\mathrm{FeNO}$ ) are positively correlated with sputum and biopsy eosinophils in BOBCAT [3]. Both serum periostin and FeNO trend towards a positive correlation with biopsy neutrophils ( $\mathrm{rs}=0.25, \mathrm{p}=0.06$ for periostin; $\mathrm{rs}=0.23, \mathrm{p}=0.08$ for $\mathrm{FeNO}$ ), and exhibit significantly negative correlations with sputum neutrophils ( $\mathrm{rs}=-0.31$ for periostin, $\mathrm{rS}=-0.35$ for FeNO; $\mathrm{p}<0.05$ for each). 

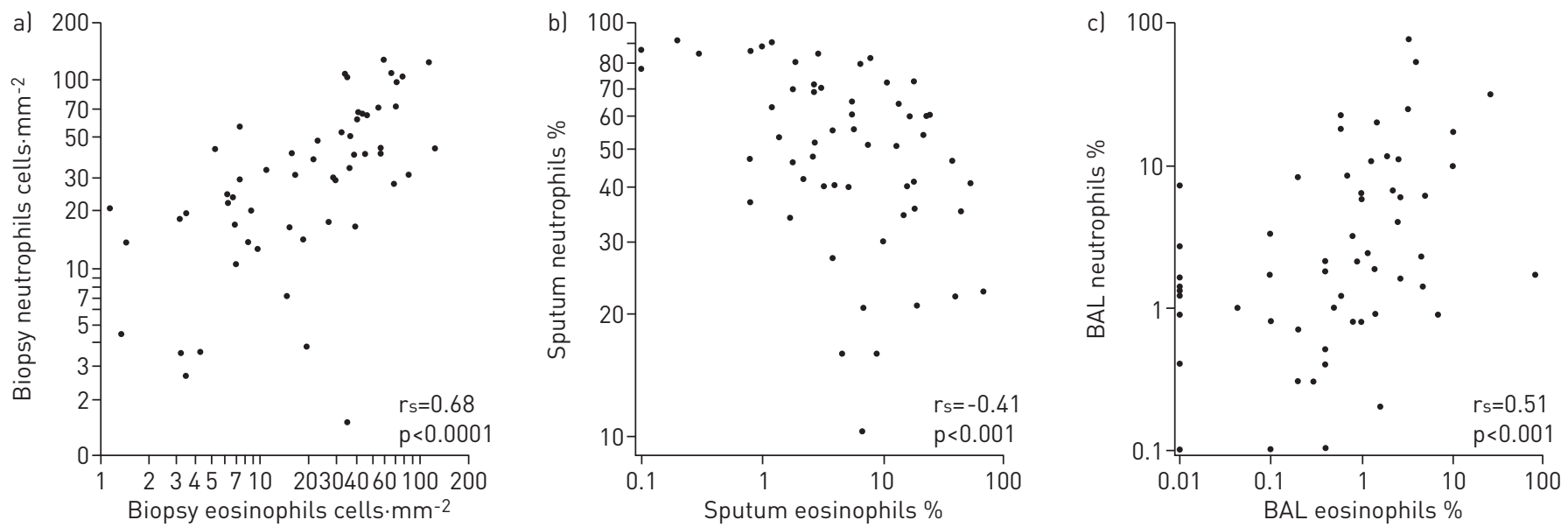

FIGURE 1 Relationships between eosinophil and neutrophil levels in each airway compartment. a) Eosinophil and neutrophil counts in endobronchial biopsy tissue; median (interquartile range) neutrophils $31(17-56)$ cells $\cdot \mathrm{mm}^{-2}$ and eosinophils $23(7-44) \mathrm{cells} \cdot \mathrm{mm}^{-2}$. Percentages of nonsquamous cells in b) induced sputum; median (IQR) neutrophils 52 (37-71)\% and eosinophils 5 (2-15)\%; and c) bronchoalveolar lavage (BAL) fluid; median (IQR) neutrophils 2 (1-8)\% and eosinophils 1 (0-3)\%. rs: Spearman's rank correlation.

None of the airway measures, nor blood biomarkers, exhibited significant correlations with lung function as assessed by FEV1 or asthma control as assessed by the ACQ (not shown).

Significantly elevated airway neutrophils are not typically observed in mild-moderate asthma patients not taking ICS or on low-dose ICS, but are often seen in severe asthma patients on high-dose steroids $[7,8]$, suggesting that chronic ICS treatment is related to elevated airway neutrophils. Our observations add to these findings by showing that, in patients with moderate-severe asthma, mucosal neutrophils are particularly elevated in subjects with tissue eosinophilia despite ICS treatment, and this relationship scales continuously.

Although eosinophil and neutrophil percentages in sputum are measured on a continuous scale, a desire to classify asthma into discrete subsets has yielded "cut-offs" for eosinophilia around 2-3\%, whereas cut-offs for neutrophilia range from $40 \%$ to $>60 \%[1,2,7,9,10]$. This is because eosinophils are typically absent in nonasthmatic subjects, while a substantial proportion of sputum cells are neutrophils even in healthy subjects [9]. As a considerable fraction of sputum is composed of neutrophils, an increase in the proportion of another cell subset may come at the expense of neutrophil percentage, setting up a propensity for inherently negative correlations between proportions of the two cell types. While changes in the relative proportion of a minor component of the cellular content of a sample (e.g. sputum eosinophils) may be informative, caution must be exercised in interpreting the relevance of changes in the relative proportion of a component representing the majority or significant plurality of the cellular content of a sample (e.g. sputum neutrophils). Consistent with these considerations, sputum eosinophils exhibited substantial proportional variability, but remained a minor component of sputum cells and correlated with tissue and BAL eosinophils, serum periostin, blood eosinophils and FeNO [3]. Sputum neutrophils exhibited less proportional variability in this study and largely appeared to vary as a negative function of sputum eosinophil percentage (fig. 1).

The weakness of the positive correlations observed between eosinophils across sputum, biopsies and BAL may be due to several factors, including: 1) the three compartments sample different regions of the airways; 2) factors mediating transmigration of granulocytes through bronchial mucosal tissue into the airway lumen are poorly understood; 3) the techniques for enumerating and reporting granulocytes in each compartment vary; and 4) each assessment in this study is cross-sectional, with sputum sampling taking place close to, but not contemporaneous with bronchoscopy. Biomarkers that integrate total inflammatory burden systemically may present a means of circumventing the variability across airway compartments. As previously reported for the BOBCAT cohort, patients with low eosinophil levels in both sputum and bronchial tissue had the lowest serum periostin levels, while subjects with elevated eosinophils in either one or the other compartment had intermediate periostin levels, and subjects with elevated eosinophils in both sputum and bronchial tissue had the highest periostin levels [3]. Therefore, sampling multiple airway compartments and/or systemic biomarkers may be a more sensitive means to ascertain whether a given asthma patient has eosinophilic airway inflammation that may be missed by sampling only one compartment.

BOBCAT represents a large, well-characterised cohort of moderate-severe asthma patients resistant to highdose ICS with intensive airway sampling. The key findings from this study are as follows. 1) Sputum, biopsy 
and BAL eosinophils are imperfectly but positively correlated, suggesting that eosinophilia in each of those compartments may be informative in moderate-severe asthma. 2) While neutrophils are positively correlated with eosinophils in biopsies and BAL, they are negatively correlated with eosinophils in sputum. Whether this is a technical artefact or a biologically relevant finding is unclear, but given recent attention devoted to "neutrophilic" phenotypes in severe asthma, this should be investigated further, particularly in the context of interventional studies of ICS and new investigational asthma therapies. 3) Eosinophil and neutrophil levels relate to each other on a continuous scale, which suggests that defining discrete cut-offs for airway phenotypes should be undertaken with caution. Future studies should also examine the longitudinal variability of granulocytic infiltration, as well as the ability of these phenotypic variables to predict outcomes in interventional studies. misleading http://ow.ly/qmNHw

Joseph R. Arron ${ }^{1}$, David F. Choy ${ }^{1}$, Michel Laviolette ${ }^{2}$, Steven G. Kelsen ${ }^{3}$, Ammar Hatab $^{4}$, Richard Leigh ${ }^{5}$,

Neil C. Thomson ${ }^{6}$, Eugene R. Bleecker ${ }^{7}$, Ron Olivenstein ${ }^{8}$, Pedro C. Avila ${ }^{9}$, Nizar N. Jarjour ${ }^{10}$, Mario Castro ${ }^{11}$,

Gail M. Gauvreau ${ }^{12}$, James T. Good ${ }^{13}$, Joel N. Kline ${ }^{14}$, Adel Mansur ${ }^{15}$, Irvin Mayers ${ }^{16}$, Liam G. Heaney ${ }^{17}$, Qutayba Hamid $^{18}$ and Jeffrey M. Harris ${ }^{1}$

${ }^{1}$ Genentech Research and Early Development, San Francisco, CA, ${ }^{3}$ Dept of Medicine, Temple University School of Medicine, Philadelphia, PA, ${ }^{4}$ West Coast Clinical Trials LLC, Costa Mesa, CA, ${ }^{7}$ Center for Genomics and Personalized Medicine Research, Wake Forest School of Medicine, Winston-Salem, NC, ${ }^{9}$ Dept of Medicine, Northwestern University Feinberg School of Medicine, Chicago, IL, ${ }^{10}$ Dept of Medicine, University of Wisconsin-Madison, Madison, WI, ${ }^{11}$ Dept of Medicine, Washington University School of Medicine, St Louis, MO, ${ }^{13}$ Dept of Medicine, National Jewish Health, Denver, CO, and ${ }^{14}$ Dept of Internal Medicine, University of Iowa College of Medicine, Iowa City, IA, USA. ${ }^{2}$ IUCPQ, Université Laval, Quebec, QC, ${ }^{5}$ Faculty of Medicine, University of Calgary, Calgary, AB, ${ }^{8}$ Montréal Chest Institute, Montreal, QC, ${ }^{12}$ Dept of Respirology, Faculty of Health Sciences, McMaster University, Hamilton ON, ${ }^{16}$ Dept of Medicine, University of Alberta, Edmonton, $\mathrm{AB}$, and ${ }^{18}$ Meakins-Christie Laboratories, McGill University, Montreal, QC, Canada. ${ }^{6}$ Institute of Infection, Immunity, and Inflammation, University of Glasgow, Glasgow, ${ }^{15}$ Respiratory Dept, Birmingham Heartlands Hospital, Birmingham, and ${ }^{17}$ Centre for Infection and Immunity, Queens University Belfast, Belfast, UK.

Correspondence: J.M. Harris, Genentech, Inc., 1 DNA Way, MS 453b, South San Francisco, CA 94080, USA. E-mail: harris.jeffrey@gene.com

Received: July 092013 | Accepted after revision: Oct 072013 | First published online: Oct 312013

Support statement: This study was funded by Genentech, Inc.

Conflict of interest: Disclosures can be found alongside the online version of this article at www.erj.ersjournals.com

Acknowledgements: We wish to thank the patients and clinical personnel who participated in this study.

\section{References}

1 Simpson JL, Scott R, Boyle MJ, et al. Inflammatory subtypes in asthma: assessment and identification using induced sputum. Respirology 2006; 11: 54-61.

2 Hastie AT, Moore WC, Meyers DA, et al. Analyses of asthma severity phenotypes and inflammatory proteins in subjects stratified by sputum granulocytes. J Allergy Clin Immunol 2010; 125: 1028-1036.

3 Jia G, Erickson RW, Choy DF, et al. Periostin is a systemic biomarker of eosinophilic airway inflammation in asthmatic patients. J Allergy Clin Immunol 2012; 130: 647-654.

4 Juniper EF, O'Byrne PM, Guyatt GH, et al. Development and validation of a questionnaire to measure asthma control. Eur Respir J 1999; 14: 902-907.

5 Hauber HP, Gotfried M, Newman K, et al. Effect of HFA-flunisolide on peripheral lung inflammation in asthma. J Allergy Clin Immunol 2003; 112: 58-63.

6 Pin I, Gibson PG, Kolendowicz R, et al. Use of induced sputum cell counts to investigate airway inflammation in asthma. Thorax 1992; 47: 25-29.

7 McGrath KW, Icitovic N, Boushey HA, et al. A large subgroup of mild-to-moderate asthma is persistently noneosinophilic. Am J Respir Crit Care Med 2012; 185: 612-619.

8 Wenzel SE, Schwartz LB, Langmack EL, et al. Evidence that severe asthma can be divided pathologically into two inflammatory subtypes with distinct physiologic and clinical characteristics. Am J Respir Crit Care Med 1999; 160: 1001-1008.

9 Belda J, Leigh R, Parameswaran K, et al. Induced sputum cell counts in healthy adults. Am J Respir Crit Care Med 2000; 161: 475-478.

10 Haldar P, Brightling CE, Hargadon B, et al. Mepolizumab and exacerbations of refractory eosinophilic asthma. N Engl J Med 2009; 360: 973-984. 


\title{
Impact of cigarette smoking on latent tuberculosis infection: does age matter?
}

\author{
To the Editor:
}

Latent tuberculosis infection (LTBI) is defined by evidence of immunological responses by Mycobacterium tuberculosis proteins in the absence of clinical symptoms/signs of active diseases [1]. People who have increased likelihood of tuberculosis (TB) exposure and those with clinical conditions that increased their risk of progressing from LTBI to TB disease are regarded as high-risk groups for developing TB disease and should be considered for LTBI testing and treatment. Both tuberculin skin test (TST) and T-cell based interferon $-\gamma$ release assays (IGRAs) are available diagnostic tool for LTBI, but IGRAs avoid the interferences from bacille Calmette-Guérin (BCG) vaccination and non-tuberculous mycobacterium (NTM). Cigarette smoke has adverse effects in respiratory immune function and is widely reported to be associated with an increased risk of respiratory a tract infection, including TB $[2,3]$. However, only a few studies investigated the impact of smoking on LTBI, and none of these studies used IGRAs to diagnose LTBI $[4,5]$.

The European Respiratory Journal recently published a perspective review focused on the role of diagnosis and treatment of LTBI to improve TB control and eventually TB elimination [6]. To eliminate TB on a global scale the identification and sterilisation of latently infected individuals, especially those in high-risk groups, is of paramount importance. Concerning the close correlation between cigarette smoking and active $\mathrm{TB}$, the association between LTBI and smoking deserves further clarification with IGRAs as a diagnostic tool.

To elucidate the issue, we enrolled inpatients and outpatients who were considered at risk for LTBI and progression to active TB disease from January, 2011 to March, 2012 in a tertiary medical centre in Taiwan. The high-risk individuals enrolled in the present study included people with active TB contact, healthcare workers, and patients with: malignancy, diabetes mellitus, end-stage renal disease, liver cirrhosis, post-organ transplantation, autoimmune diseases, and fibrocalcified lesions suggestive of prior $\mathrm{TB}$ on a chest radiograph. Enrolled patients who were under anti-TB treatment or diagnosed with active TB within 1 month of enrolment were excluded. The status of cigarette smoking was collected at the enrolment interview and enrolled patients were divided into ex-smokers, current smokers and never-smokers. The presence of LTBI in enrolled patients was determined by the results of IGRA. The IGRA was performed with QuantiFERON-TB Gold In-Tube (QFT-GIT; Qiagen, Hilden, Germany). The test results were determined as negative, indeterminate, or positive (cut-off at $0.35 \mathrm{IU} \cdot \mathrm{mL}^{-1}$ ) according to the manufacturer's software. Univariate and multivariate analysis were performed to determine the clinical factors associated with LTBI. Statistical analyses were carried out using SPSS version 17.0 software (SPSS, Inc., Chicago, IL, USA).

During the study period, a total of 955 high-risk individuals were enrolled, 80 of which had indeterminate QFT-GIT results. Ultimately, 875 patients with determinate QFT-GIT results, including 147 (16.8\%) former smokers, 125 (14.3\%) current smokers, and 603 (68.9\%) never-smokers, were included for analysis. The proportions of LTBI cases in ex-smokers, current smokers and never-smokers were 58 (39.5\%) out of 147, $53(42.4 \%)$ out of 125 , and $151(25 \%)$ out of 603 , respectively, which was significantly lower in the neversmokers $(\mathrm{p}<0.001)$. As compared with never-smokers, ex-smokers and current smokers were older $(\mathrm{p}<0.001)$, more likely to be male $(\mathrm{p}<0.001)$, have chronic obstructive pulmonary disease $(\mathrm{p}<0.001)$, have some malignancy $(\mathrm{p}<0.001)$, and have fibrocalcified lesions on chest radiographs $(\mathrm{p}<0.001)$. Ex-smokers and current smokers were less likely to: have a TB-contact history $(p=0.004)$, have received a BCG vaccination $(\mathrm{p}<0.001)$, have certain autoimmune disorders $(\mathrm{p}<0.001)$, and to be healthcare workers $(\mathrm{p}<0.001)$ when compared with never-smokers. In multivariate analysis, both ex-smoking (OR 1.64, 95\% CI 1.00-2.68) and current smoking (OR 1.88, 95\% CI 1.16-3.03) were independent factors associated with LTBI. Other significant factors included increased age (for ex-smoking OR 1.01, 95\% CI 1.00-1.03; for current smoking OR 1.02, 95\% CI 1.01-1.03) and COPD (for current smoking OR 3.15, 95\% CI 1.03-9.69).

We further analysed the impact of smoking in patients with different ages. The proportions of LTBI among various age groups are shown in figure 1. In ever-smokers, the proportions of LTBI dramatically escalated as age increased but declined gradually after the age of 75 years. In never-smokers, the proportions of LTBI also escalated with increasing age but were similar in patients $>45$ years. The odds ratio of smoking for LTBI in each age group is also shown in figure 1 . We found that the odds ratio was higher in elderly patients than those with a younger age, and the highest risk of smoking for LTBI was noted in patients aged from 


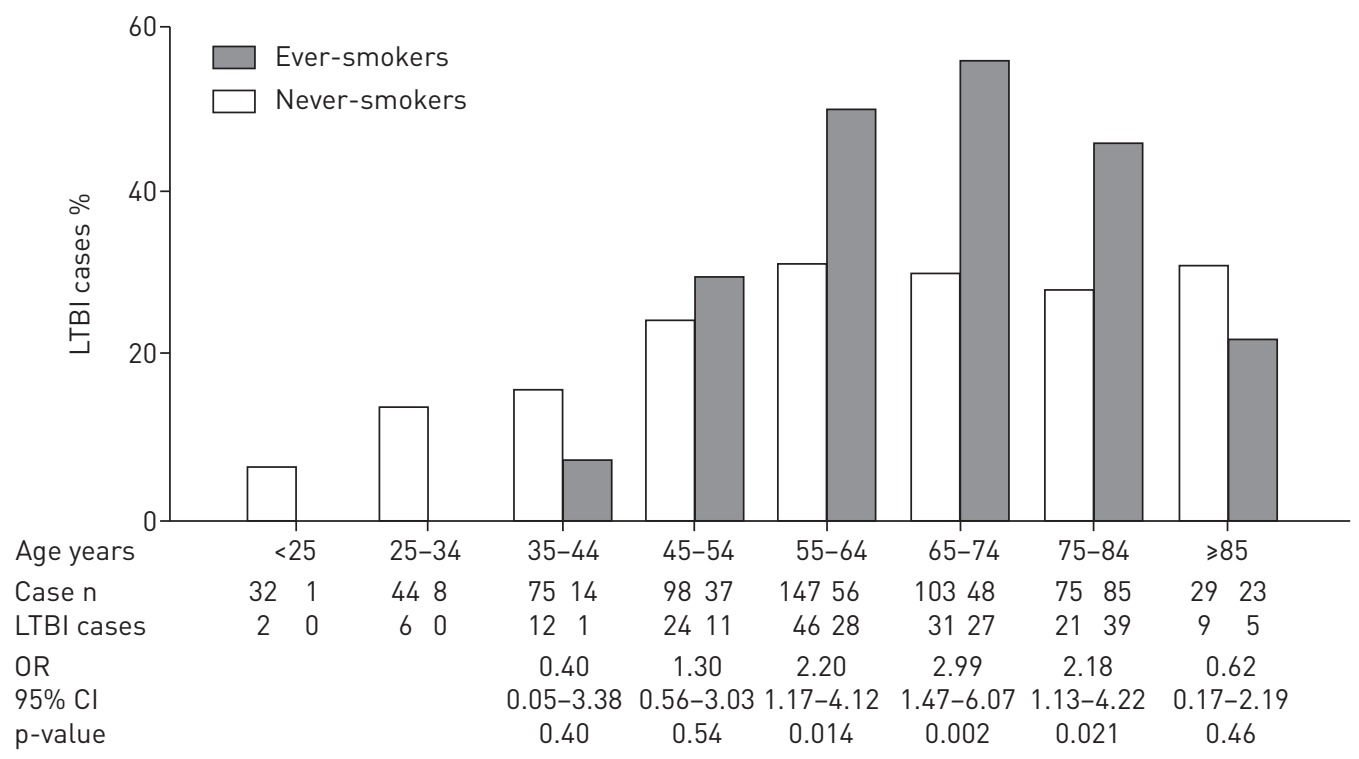

FIGURE 1 Proportions of latent tuberculosis infection (LTBI) among high-risk individuals, stratified by age group and smoking status. Case numbers of overall population and LTBI are presented, and odds ratio of smoking in each age group is analysed using univariate analysis.

65-74 years (OR 2.99, 95\% CI 1.47-6.07). Interestingly, the odds ratio rapidly declined in the population aged $>85$ years.

The occurrences of active TB were documented in eight (1.33\%) out of 603 never-smokers and seven (2.57\%) out of 272 ever-smokers during the 1-year follow-up period, with the log rank p-value of 0.19 using Kaplan-Meier analysis. 12 of them were documented with LTBI when at enrolment.

Despite extensive studies investigating the association between smoking and active TB, reports evaluating the impact of smoking on LTBI are relatively scarce $[4,5]$. To our knowledge, this is the first study that used IGRA to analyse the association between smoking and LTBI. We clearly identified smoking as an independent risk factor for LTBI, both in ex-smokers and current smokers. As compared with previous studies undertaken in TB-endemic areas with TST, our results should be more specific without the potential interferences from BCG vaccination and NTM, especially in TB endemic areas where the BCG vaccination was widely adopted.

The immunological effects of cigarette smoke had been reported in previous studies. Smoke-exposed mice had fewer cytokine-producing macrophages with diminished influx of interferon- $\gamma$ producing effector Tcells in the lung than mice without smoke exposure [7]. These adverse effects of cigarette smoke in pulmonary immunity contribute to increased incidence of active TB and LTBI. Some clinical studies speculated that the impaired immunity associated with smoking may lower the positive rates of IGRA in active TB cases and HIV patients with LTBI $[8,9]$. The controversial findings between the present study and previous reports deserve further clarification in different study populations.

We also found the impact of smoking on LTBI varied with age. Patients with an increased age were more vulnerable to the negative impact of smoking in the occurrence of LTBI. The aged population probably had longer duration and/or higher intensity in cigarette smoking. The immune-suppressive effects of cigarette smoke are probably more prominent in an aged population. The lung structure impairment related to increased intensity of cigarette exposure in aged patients may also increase their susceptibilities to $M$. tuberculosis TB. Therefore, the different impact of intensity and duration of smoking on LTBI deserves further clarification.

The World Health Organization published a monograph to announce the integration of tobacco control into TB programmes in 2007 [10]. However, the importance of smoking in LTBI screening and treatment is so far underestimated. Our findings enhance the evidences that cigarette smoking was an important risk factor associated with a higher prevalence of LTBI. Current and former smokers should be considered as a high-risk population for LTBI and potential candidates for LTBI prophylaxis treatment. Meanwhile, smoking cessation is definitely an important measure to decrease the prevalence of LTBI both in low- and 
high-TB prevalence areas. To eliminate TB in the future, the importance of anti-smoking campaigns should not be overlooked.

0 @ERSpublications

Current and ex-smoking is associated with increased risk of LTBI in high-risk individuals, especially in aged populations http://ow.ly/q5FTK

Jia-Yih Feng ${ }^{1,2}$, Shiang-Fen Huang ${ }^{1,3}$, Wen-Ying Ting ${ }^{1}$, Ming-Che Lee ${ }^{1}$, Yu-Chun Chen ${ }^{3,4}$, Yung-Yang Lin ${ }^{2,5,6}$, $\mathrm{Yu}$-Chin Lee ${ }^{1,3}$ and Wei-Juin $\mathrm{Su}^{1,3}$

${ }^{1}$ Dept of Chest Medicine, Taipei Veterans General Hospital, Taipei, ${ }^{2}$ Institute of Clinical Medicine, School of Medicine, National Yang-Ming University, Taipei, ${ }^{3}$ School of Medicine, National Yang-Ming University, Taipei, ${ }^{4}$ Dept of Medical Research and Education, National Yang-Ming University Hospital, Taipei, ${ }^{5}$ Institute of Brain Science, National YangMing University, Taipei, and ${ }^{6}$ Laboratory of Neurophysiology and Dept of Neurology, Taipei Veterans General Hospital, Taipei, Taiwan, R.O.C.

Correspondence: W-J. Su, Dept of Chest Medicine, Taipei Veterans General Hospital, \#201, Section 2, Shih-Pai Road, Taipei 112, Taiwan, R.O.C. E-mail: wjsu@vghtpe.gov.tw

Received: June 112013 | Accepted after revision: Sept 022013 | First published online: Sept 262013

Support statement: This study was funded by the Institute for Biotechnology and Medicine Industry, Taiwan, R.O.C., and Taipei Veterans General Hospital (V100A-002, V101B-027 and V102B-030).

Conflict of interest: None declared.

\section{References}

1 Barry CE 3rd, Boshoff HI, Dartois V, et al. The spectrum of latent tuberculosis: rethinking the biology and intervention strategies. Nat Rev Microbiol 2009; 7: 845-855.

2 Garmendia J, Morey P, Bengoechea JA. Impact of cigarette smoke exposure on host-bacterial pathogen interactions. Eur Respir J 2012; 39: 467-477.

3 Leung CC, Yew WW, Law WS, et al. Smoking and tuberculosis among silicotic patients. Eur Respir J 2007; 29: 745-750.

4 den Boon S, van Lill SWP, Borgdorff M, et al. Association between smoking and tuberculosis infection: a population survey in a high tuberculosis incidence area. Thorax 2005; 60: 555-557.

5 Horne DJ, Campo M, Ortiz JR, et al. Association between smoking and latent tuberculosis in the U.S. population: an analysis of the National Health and Nutrition Examination Survey. PLoS One 2012; 7: e49050.

6 Diel R, Loddenkemper R, Zellweger JP, et al. Old ideas to innovate tuberculosis control: preventive treatment to achieve elimination. Eur Respir J 2013; 42: 785-801.

7 Shang S, Ordway D, Henao-Tamayo M, et al. Cigarette smoke increases susceptibility to tuberculosis - evidence from in vivo and in vitro models. J Infect Dis 2011; 203: 1240-1248.

8 Oni T, Gideon HP, Bangani N, et al. Smoking, BCG and employment and the risk of tuberculosis infection in HIVinfected persons in South Africa. PLoS One 2012; 7: e47072.

9 Aabye MG, Hermansen TS, Ruhwald M, et al. Negative effect of smoking on the performance of the QuantiFERON TB gold in tube test. BMC Infect Dis 2012; 12: 379 .

10 World Health Organization. The International Union Against Tuberculosis and Lung Disease. A WHO/The Union Monograph on TB and Tobacco Control. Joining Efforts to Control Two Related Global Epidemics. Geneva, WHO, 2007; p. 9.

\section{A tale of two settings: the role of the Beijing genotype in the epidemiology of multidrug-resistant tuberculosis}

\section{To the Editor:}

Described as a "template for success" by НАNEKOM et al. [1] the Beijing genotype of Mycobacterium tuberculosis has been associated with hypervirulence, drug resistance, evasion of the bacille Calmette-Guérin (BCG) vaccine and differential immunoregulation [1]. The genotype is itself diverse; accordingly the fact that specific traits have been associated with Beijing only in certain settings may be explained by the variation in the subtypes that predominate in each. 
We sought to explore the role that the Beijing genotype plays in the epidemiology of multidrug-resistant (MDR) tuberculosis (TB) in two very different epidemiological settings: England and Peru. In the UK nearly three quarters of TB disease occurs amongst migrants, which may result from latent M. tuberculosis infections acquired from high-burden countries of origin. Thus the distribution of lineages in the UK reflects a composite of the circulating strains in other nations. Whilst increasing levels of MDR-TB in the UK have triggered understandable concern, only a small fraction of cases of TB are MDR (in 2011 this was $1.0 \%$ ) [2]. In contrast, the Peruvian TB epidemic is relatively "home-grown" and the prevalence of drug resistance is greater (in $20115.3 \%$ were MDR-TB) [2]. Our objectives were thus: 1) identify any association between the Beijing genotype and MDR in these two settings, and 2) to examine if trends in Beijing might explain changes in the prevalence of MDR-TB over time.

Four datasets were used during this study: two English studies and two Peruvian studies.

The English datasets were taken from Public Health England's Enhanced Tuberculosis Surveillance System and Mycobacterial Surveillance Network and were referred to as England 1 and England 2. England 1 consisted of 8859 individuals diagnosed and 24-loci MIRU-VNTR (mycobacterial interspersed repetitive units variable number tandem repeats) strain-typed between 2010 and 2011. Of these, 4661 had at least first-line drug sensitivity data, a minimal 12-loci predefined profile [3] and were pulmonary. England 2 consisted of 189 MDR-TB cases from 2004 to 2007, which had undergone 24-loci typing. 117 were pulmonary and has at least the minimal standard 12-loci profile. 24-loci typing was not available for nonMDR cases during this period. Susceptibility to rifampicin, isoniazid, ethambutol, pyrazinamide, streptomycin, amikacin, kanamycin, capreomycin, azithromycin, clarithromycin, ciprofloxacin, ofloxacin,

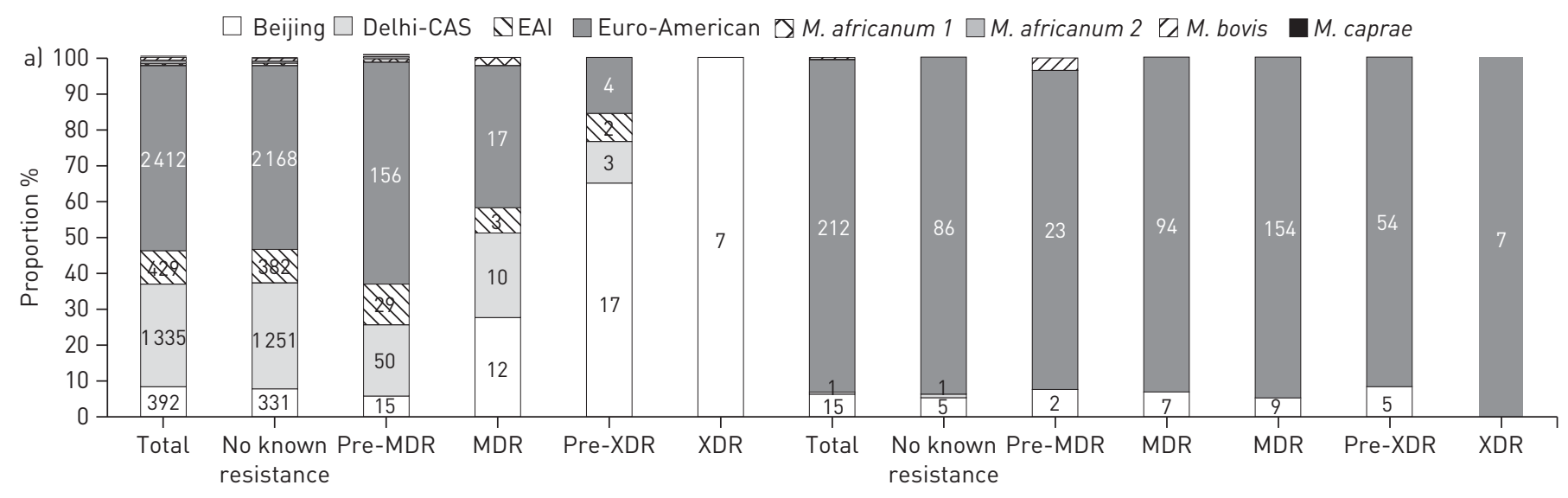

Mutually exclusive resistance categories

England 1

Peru 1

Peru 2

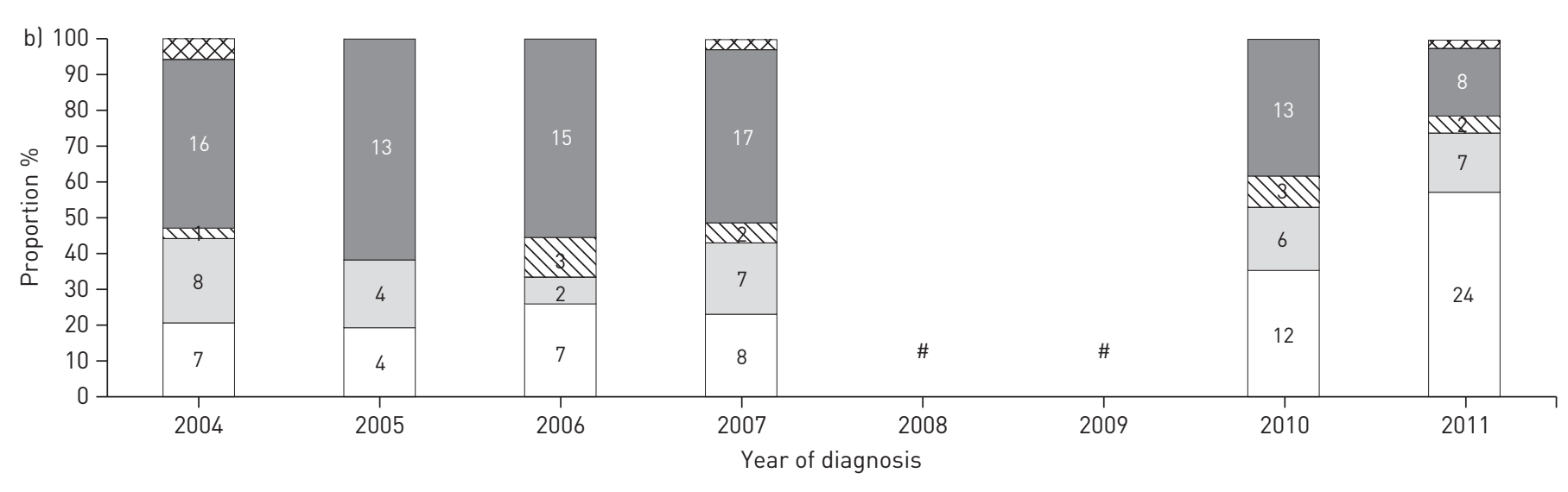

England 1

England 2

FIGURE 1 a) The Beijing genotype is enriched with increasing drug resistance in English (England 1) (ptrend $<0.005$ Bejing versus non-Beijing), but not Peruvian (Peru 1 and Peru 2) pulmonary tuberculosis (TB) cases. b) Lineages of the English pulmonary multidrug resistant (MDR) TB cases (England 1 and England 2), presented by year of diagnosis. The proportion of cases associated with the Beijing genotype may be increasing with time in the latter years. CAS: central Asian; M.: Mycobacterium; EAI: East-African Indian; XDR: extensively drug resistant. *: 24-loci MIRU-VNTR typing was not undertaken between 2008 and 2009. 
moxifloxacin, prothionamide, ethionamide, cycloserine, 4-aminosalicylic acid, linezolid, clofazamine and rifabutin had been tested using the proportion or resistance ratio methods.

The Peruvian datasets were referred to as Peru 1 and Peru 2. Peru 1 consisted of 323 sputum-positive cases of pulmonary TB, recruited from clinical facilities in Lima between 2004 and 2006 and extracted from TAYPE et al. [4]. 229 had been tested for sensitivity to rifampicin, isoniazid, ethambutol and streptomycin, of which $101(44.1 \%)$ were MDR; we note that this figure is much higher than the overall proportion of MDR in this setting [2], suggesting that drug susceptibility testing was prioritised for those at highest risk of MDR, consistent with previous Peruvian testing policies. All cases had a complete standard 12-loci profile. Peru 2 consisted of 230 cases of pulmonary MDR-TB identified in Lima between 1996 and 2004 [5, 6]. Isolates underwent drug susceptibility testing to rifampicin, isoniazid, ethambutol, pyrazinamide, streptomycin, kanamycin, capreomycin, ethionamide, 4-aminosalicylic acid and levofloxacin. One individual lacking a complete standard 12-loci profile was excluded, leaving 229.

MIRU-VNTRplus was utilised to designate lineages for all four datasets to ensure comparability [7]. Substantial differences in the lineage and sublineage profiles of English (England 1, 4661 individuals) and Peruvian (Peru 1, 229 individuals) pulmonary TB cases were apparent (Chi-squared test of independence pvalue $<0.005)$. The vast majority of Peruvian cases were of the Euro-American lineage $(212(92.6 \%)$ out of 229), with Latin American-Mediterranean (LAM) the majority sublineage (102 (48.1\%) out of 212). The remainder of cases were nearly all Beijing (15 (6.6\%) out of 229). In contrast, English cases comprised of a variety of lineages consistent with their multinational origins: Euro-American $2412(51.8 \%)$ out of 4661, Delhi-CAS (central-Asia) 1335 (28.6\%) out of 4661, East African Indian (EAI) 429 (9.2\%) out of 4661, Beijing 392 (8.3\%) out of 4661. The Euro-American sublineages observed were also diverse.

Restricting our analysis to MDR cases revealed very different profiles between Peru and England ( $\mathrm{p}$-value $<0.005)$. Peruvian cases (Peru 2, 229 individuals) were dominated by the Euro-American lineage (215 (93.9\%) out of 229), of which 121 (56.3\%) out of 215 was the LAM sublineage. Regression modelling of Peru 1 to explore the relationship between MDR and M. tuberculosis lineage showed no association (Beijing crude OR (95\% CI) 1.10 (0.38-3.14), Wald p-value 0.86; Euro-American baseline). In stark contrast to the overall distribution of lineages, among English MDR cases (England 1) the proportion Beijing increased to 36 (47.4\%) out of 76. Regression modelling revealed a strong positive association between Beijing and MDR among English pulmonary cases, (crude OR 11.51 (6.65-19.95), p<0.005; adjusted OR 9.53 (4.87-18.65), $\mathrm{p}<0.005$; Euro-American baseline and England 1 used for both models; models excluded non- $M$. tuberculosis cases and those with missing data). Age, sex, a composite social risk factor variable (homelessness, imprisonment, drug/alcohol abuse), country of birth, ethnic group and previous diagnosis were adjusted for in the latter; this could not be done for Peru 1 as these variables were not available.

We also tested whether Beijing was enriched among strains with resistance to a greater number of drugs (fig. 1a). Pre-MDR-TB (pre-MDR) was defined as resistance to either, but not both, isoniazid and rifampicin. Pre-extensively drug resistant (pre-XDR) cases were MDR plus resistant to either a fluoroquinolone or a second-line injectable. Beijing was enriched from pre-MDR to XDR cases in the English data (Pearson's correlation coefficient (ptrend) Beijing versus non-Beijing $\mathrm{p}<0.005$; England 1 ). This could be specifically observed among non-UK born, but not UK born, cases (data not shown). No trend was seen in the Peruvian data (ptrend=0.76; Peru 1 and Peru2). Further analysis to determine whether the association has changed over time indicated a recent increase in the proportion of cases caused by Beijing (ptrend $<0.005$; England 1 and England 2) (fig. 1b).

Our analysis suggests that the Beijing genotype plays a different role in the current epidemiology of MDRTB in England and Peru. In England, the observed association likely reflects the rising importation of resistant strains, rather than the development of resistance within the UK. In Peru there is likely to be a similarly high prevalence of MDR among all genotypes, potentially explaining why we did not observe an association. Interestingly, a recent paper by IШАмото et al. [8] suggests that the overall prevalence of Beijing has increased in Peru over the last decade, but the proportion of Beijing strains that are MDR has decreased.

We utilised available datasets from studies undertaken at different times with variable designs, thus some interpretative caution is required. For example, Peru 2 recruited from households with more than one pulmonary MDR case, and thus could have oversampled clustered cases. However, the similarity of the lineage profiles of Peru 2 with Peru 1, which was not collected through such means, suggests this effect is limited. English cases were tested for resistance to a wider range of drugs than the Peruvian cases, thus the "no known resistance" category is not strictly comparable between the two datasets. Nevertheless, only 1.3\% of the England 1 dataset was resistant to drugs not tested within the Peru 1 dataset.

Molecular epidemiological surveillance and addressing and preventing resistance have been highlighted as vital for TB elimination by the European Respiratory Society $[9,10]$. We demonstrate within this study how 
the tools of the former could be vital to aid the latter: within Peru the MDR epidemic could worsen should fitter drug resistant Beijing strains evolve or enter the country and within the UK where the lineage profile is highly dynamic.

Contrasting relationship between the Beijing genotype of Mycobacterium tuberculosis and MDR-TB in England versus Peru. http://ow.ly/qjxTG

Helen R. Stagg ${ }^{1,2,3}$, Ted Cohen ${ }^{3,4}$, Mercedes C. Becerra ${ }^{5}$, Laura F. Anderson ${ }^{2}$ and Ibrahim Abubakar ${ }^{1,2}$

${ }^{1}$ Research Dept of Infection and Population Health, University College London, and ${ }^{2}$ TB Section, Dept of Respiratory Diseases, Public Health England, London, UK. ${ }^{3}$ Division of Global Health Equity, Brigham and Women's Hospital, Boston, MA, ${ }^{4}$ Dept of Epidemiology, Harvard School of Public Health, Boston, MA, and ${ }^{5}$ Dept of Global Health and Social Medicine, Harvard Medical School, Boston, MA, USA.

Correspondence: H.R. Stagg, Research Dept of Infection and Population Health, University College London, 4th floor, Mortimer Market Centre, Mortimer Market, London, WC1E 6JB, UK. E-mail: h.stagg@ucl.ac.uk

Received: July 032013 | Accepted after revision: Aug 312013 | First published online: Sept 132013

Support statement: H.R. Stagg was supported by a European Respiratory Society Fellowship STRTF 24-2011. The strain typing of the English datasets was funded by Public Health England's Strain Typing Project. I. Abubakar was funded through an NIHR Senior Research Fellowship NIHR-2011-04-001.

Conflict of interest: Disclosures can be found alongside the online version of this article at www.erj.ersjournals.com

Acknowledgements: We acknowledge our utilisation of the published data of TAYPE et al. [4]. Our study made use of the MIRU-VNTRplus database website (www.miru-vntrplus.org) developed by D. Harmsen, S. Niemann, P. Supply and T. Weniger.

\section{References}

1 Hanekom M, Gey van Pittius NC, McEvoy C, et al. Mycobacterium tuberculosis Beijing genotype: a template for success. Tuberculosis (Edinb) 2011; 91: 510-523.

World Health Organization. Global Tuberculosis Control Report. Geneva, World Health Organization, 2012.

3 Savine E, Warren RM, van der Spuy GD, et al. Stability of variable-number tandem repeats of mycobacterial interspersed repetitive units from 12 loci in serial isolates of Mycobacterium tuberculosis. J Clin Microbiol 2002; 40: 4561-4566.

4 Taype CA, Agapito JC, Accinelli RA, et al. Genetic diversity, population structure and drug resistance of Mycobacterium tuberculosis in Peru. Infect Genet Evol 2012; 12: 577-585.

5 Becerra MC, Appleton SC, Franke MF, et al. Tuberculosis burden in households of patients with multidrugresistant and extensively drug-resistant tuberculosis: a retrospective cohort study. Lancet 2011; 377: 147-152.

6 Mitnick C, Bayona J, Palacios E, et al. Community-based therapy for multidrug-resistant tuberculosis in Lima, Peru. N Engl J Med 2003; 348: 119-128.

7 Allix-Béguec C, Harmsen D, Weniger T, et al. Evaluation and strategy for use of MIRU-VNTRplus, a multifunctional database for online analysis of genotyping data and phylogenetic identification of Mycobacterium tuberculosis complex isolates. J Clin Microbiol 2008; 46: 2692-2699.

8 Iwamoto T, Grandjean L, Arikawa K, et al. Genetic diversity and transmission characteristics of Beijing family strains of Mycobacterium tuberculosis in Peru. PLoS One 2012; 7: e49651.

9 Blasi F, Reichman LB, Migliori GB. Presenting the European Forum for TB Innovation: innovative thinking in progressing towards TB elimination in Europe. Eur Respir J 2012; 40: 806-808.

10 Diel R, Loddenkemper R, Zellweger JP, et al. Old ideas to innovate tuberculosis control: preventive treatment to achieve elimination. Eur Respir J 2013; 42: 785-801.

\section{Helicobacter pylori seroprevalence in patients with idiopathic pulmonary fibrosis}

\section{To the Editor:}

In 2007, the European Respiratory Journal published a letter by IBRAHIM [1] in which it was suggested that micro-aspiration of Helicobacter pylori from gastric juice secondary to gastro-oesophageal reflux disease (GORD) may cause or contribute to the development of idiopathic pulmonary fibrosis (IPF) through recurrent lung insult. This hypothesis was derived from the observation that GORD is common in IPF patients $[2,3]$, while in the general population the prevalence of GORD exceeds that of IPF. IBRAHIM [1] 
postulated that only a small proportion of subjects with GORD develop IPF, probably those with $H$. pylori in their gastric juice. This theory has not yet been investigated and no data is available in the literature on the relationship between $H$. pylori infection and IPF.

H. pylori is a Gram-negative, spiral bacterium. There are two major groups of H. pylori microorganisms: type I, which expresses the vacuolating cytotoxin VacA and the cytotoxin-associated gene CagA and is responsible for mucosal damage, inducing local inflammatory response; and type II, which expresses neither of these proteins and does not determine any injury [4]. Type I strains, which have the chromosomal insertion called Cag, are endowed with increased inflammatory function that may determine further augmentation of local and systemic cytokines [5].

H. pylori infection is very common all over the world and does not seem to have sex or racial prevalence. CagA H. pylori strains play a role in gastroduodenal ulcers and the development of gastric cancer. H. pylori infection was recently demonstrated to protect against gastro-oesophageal reflux disease and reflux esophagitis [6], to be associated with several extragastric disorders [7] and to play a role in respiratory disorders, such as chronic bronchitis and lung cancer $[4,8,9]$. A protective function in bronchial asthma, rhinitis and T-helper cell type 2 (Th-2) driven disorders in general has been proposed [10].

In order to contribute to the analysis of $H$. pylori infection in IPF, we designed a pilot study that included serological analysis. We evaluated serum-prevalence of total anti-H. pylori antibodies (anti-HP Ab) and anti-CagA antibodies (anti-CagA Ab) in a population of IPF patients. 45 patients with IPF (33 males, mean age $66.2 \pm 10.2$ years, 12 out of 45 had a histologically confirmed diagnosis and 33 out of 45 had a definite high-resolution computed tomography (HRCT) diagnosis) monitored at the Regional Referral Center for Sarcoidosis and other Interstitial Lung Diseases at Siena University (Siena, Italy), were enrolled, consecutively, in the study. Inclusion criteria were: 1 ) a documented history of IPF of $>1$ year; 2) diagnosis of IPF performed according to American Thoracic Society/European Respiratory Society/Japanese Respiratory Society/Asociacion Latinoamericana del Tórax guidelines [11]; 3) regular follow-up with chest HRCT (almost one a year); 4) pulmonary function tests (PFTs) performed according to guidelines (almost every 6 months). Patients with malignancies and pulmonary hypertension were excluded.

The results were compared with a population-based sample of 797 age- and sex-matched controls with a comparable socioeconomic background (Siena Osteoporosis Cohort) [12], obtained from primary care registers of Siena residents.

All participants gave their written informed consent to the study that was approved by the local ethics committee.

Serum IgG antibodies to $H$. pylori were analysed in all patients and controls by ELISA having a sensitivity and specificity of 96\% (Diesse Diagnostica Senese, Monteriggioni, Italy); serum anti-CagA IgG antibodies were determined by ELISA with a sensitivity of $95 \%$ and a specificity of $90 \%$ (CagA-IgG; Genesis Diagnostics Ltd., Cambridgeshire, UK). Cut-off values of $6.2 \mathrm{IU} \cdot \mathrm{mL}^{-1}$ for anti-HP antibodies and $5.5 \mathrm{IU} \cdot \mathrm{mL}^{-1}$ for anti-CagA antibodies were based on previous studies [5].

Statistical analysis was performed using GraphPad Prism version 4.0 for Macintosh, $\mathrm{p}<0.05$ was considered significant. Differences between the two groups were studied by Mann-Whitney test; analysis of variance was done by Kruskal-Wallis test, while Fisher's exact test or the Chi-square test was applied to evaluate prevalence by contingency tables. All data was expressed as mean $\pm \mathrm{SD}$.

Table 1 reports the prevalence of anti-HP Ab and anti-CagA Ab in the IPF population together with demographic data, functional test parameters at baseline and at 1-year follow-up, bronchoalveolar lavage (BAL) cell pattern, CD8:CD4 ratio, and 6-month and 1-year mortality rates.

Serum IgG antibodies against $H$. pylori proved positive in 18 out of 45 patients with IPF (prevalence $40 \%$ ), 10 of whom were also positive to anti-CagA serum antibodies (55\%). The population-based control group showed a $H$. pylori infection prevalence of $51.4 \%$ (410 out of 797), 206 of whom were positive to anti-CagA serum antibodies $(50.2 \%)$.

Prevalence of $H$. pylori infection was not significantly different in patients and the general population (OR $0.62,95 \%$ CI $0.43-1.16 ; \mathrm{p}=0.16$,), and the same was found for infection by CagA-positive $H$. pylori strains (OR 1.23, 95\% CI 0.47-3.20; $\mathrm{p}=0.81$ ). No significant difference between antibody titres of anti-HP Ab $(\mathrm{p}=0.06)$ and anti-CagA $\mathrm{Ab}(\mathrm{p}=0.06)$ was found.

IPF patients testing positive for $H$. pylori showed significantly lower values of forced vital capacity (FVC), forced expiratory volume in $1 \mathrm{~s}$ (FEV1) and total lung capacity (TLC) than those testing negative for H. pylori $(\mathrm{p}=0.016, \mathrm{p}=0.026$, and $\mathrm{p}=0.037$, respectively) (table 1 ). No statistically significant difference was found for DLCO, although the number of patients unable to perform the test due to severe lung impairment 
TABLE 1 Prevalence of anti-Helicobacter pylori antibodies and anti-CagA antibodies in an idiopathic pulmonary fibrosis population along with demographic data, functional-test parameters and mortality rates

\begin{tabular}{|c|c|c|c|}
\hline & H. pylori positive & H. pylori negative & p-value \\
\hline Patients n & 18 & 27 & \\
\hline Age years & $66.8 \pm 10.8$ & $65.7 \pm 9.8$ & NS \\
\hline Males n & 16 & 19 & NS \\
\hline \multicolumn{4}{|c|}{ Pulmonary function tests } \\
\hline FVC \% pred & $53.6 \pm 20.3$ & $70.1 \pm 23.1$ & 0.016 \\
\hline FEV1 \% pred & $57.4 \pm 18.7$ & $72.1 \pm 21.9$ & 0.026 \\
\hline FEV1/FVC & $87.6 \pm 11.3$ & $82.8 \pm 7.6$ & NS \\
\hline RV \% pred & $75.1 \pm 25.9$ & $87.6 \pm 25.6$ & NS \\
\hline TLC \% pred & $61.7 \pm 16.0$ & $74.5 \pm 19.2$ & 0.037 \\
\hline DLCo \% pred & $42.0 \pm 17.1$ & $42.2 \pm 20.1$ & NS \\
\hline Kco \% pred & $71.1 \pm 23.4$ & $66.6 \pm 20.4$ & NS \\
\hline$\Delta \mathrm{FVC} \%$ & -11 & -5.3 & 0.05 \\
\hline$\Delta D$ LCO $\%$ & -12.6 & -6 & NS \\
\hline \multicolumn{4}{|l|}{ Mortality \% } \\
\hline At 6 months & 61.1 & 28 & 0.05 \\
\hline At 1 year & 66.6 & 37 & NS \\
\hline
\end{tabular}

Data are expressed as mean \pm SD, unless otherwise stated. FVC: forced vital capacity; FEV1: forced expiratory volume in $1 \mathrm{~s}$; RV: residual volume; TLC: total lung capacity; DLCO: diffusing capacity of the lung for carbon monoxide; Kco: transfer coefficient of the lung for carbon monoxide; $\triangle \mathrm{FVC}$ : decline rate expressed as $\%$ of FVC; $\triangle D L C O$ : decline rate expressed as $\%$ of DLCO; NS: not significant.

and oxygen-therapy was higher in $H$. pylori-positive (44.4\%) IPF patients than the $H$. pylori-negative IPF patients $(25.9 \%)$, suggesting their worse respiratory condition, although the difference was at the limits of significance $(\mathrm{p}=0.07)$.

6-month follow-ups recorded the death of 18 out of 45 IPF patients giving a mortality rate of $40 \%$. There was a statistically significant difference in the mortality rate of the two subgroups: $11(61.1 \%)$ out of 18 H. pylori-positive patients died versus 7 (28\%) out of the $25 \mathrm{H}$. pylori-negative patients who died (two patients were lost to follow-up) $(\mathrm{p}=0.05)$. 1-year mortality rate was higher in the $H$. pylori-positive group when compared with the $H$. pylori-negative group ( $66.6 \%$ versus $37 \%$, respectively; $\mathrm{p}>0.05$ ).

1-year follow-up PFTs were available in six $\mathrm{H}$. pylori-positive patients (12 died) and $15 \mathrm{H}$. pylori-negative patients (10 died and two were lost to follow-up), showing a significant decrement in FVC in both groups $(\mathrm{p}<0.05)$. The decrement was significantly greater in $H$. pylori-positive patients when compared with the H. pylori-negative patients $(-11 \%$ versus $-5.3 \%$, respectively; $\mathrm{p}<0.05)$. Follow-up of DLCO was available in only four $\mathrm{H}$. pylori-positive patients and $12 \mathrm{H}$. pylori-negative patients and the decrease was not significant in either group $(-12.6 \%$ versus $-6 \%$, respectively; $\mathrm{p}>0.05)$.

No statistical differences in therapy and in BAL features were found between $H$. pylori-positive and H. pylori-negative IPF patients (data not shown).

No differences were observed in the prevalence of $H$. pylori-CagA positivity between patients and controls and no correlations were found between total anti-HP Ab or anti-CagA Ab and the clinical variables analysed.

In this pilot study, the prevalence of $H$. pylori infection in IPF patients was comparable to that of the general population and $H$. pylori antibodies were associated with a more severe disease (i.e. H. pylori-positive IPF patients had significantly lower FEV1, FVC and TLC than $H$. pylori-negative patients). Interestingly, in the literature, GORD is reported to be highly prevalent in IPF patients and has been hypothesised as a potential cause of the disease $[2,3]$. The role of $H$. pylori in the development of IPF has been postulated but never demonstrated [1].

In our study, the $H$. pylori-positive subgroup of IPF patients showed a more severe disease phenotype with higher rates of mortality and PFT decline, suggesting a possible role of this bacterium in disease progression. The theory put forward by IвRAHIM [1] is interesting and our study suggests the potential role of H. pylori in IPF progression. At a recent International Conference (6th WASOG Conference, held in Paris, June 6-7, 2013), Hogaboam [13] reported positive $H$. pylori staining in lung biopsy specimens from severe IPF patients, sustaining the involvement of $H$. pylori in this disease. No other literature is available in this field of research. 
The next step for our study will be direct determination, such as faecal $H$. pylori antigens or $H$. pylori determination in BAL (i.e. DNA PCR or culture), in a wide population of IPF patients. The effects of IPF pharmacological treatments on $H$. pylori infection remain to be established.

@ERSpublications

Helicobacter pylori infection in IPF patients is associated with higher rates of mortality and PFTs decline http://ow.ly/qKb3K

David Bennett ${ }^{1}$, Elena Bargagli ${ }^{1}$, Rosa Metella Refini ${ }^{1}$, Maria Stella Campagna ${ }^{2}$, Luigi Gennari ${ }^{2}$, Ranuccio Nuti ${ }^{2}$, Natale Figura ${ }^{2}$ and Paola Rottoli ${ }^{1}$

${ }^{1}$ Respiratory Diseases Unit, Dept of Medical and Surgical Sciences \& Neurosciences, University of Siena, Siena, and

${ }^{2}$ Internal Medicine Unit, Dept of Medical and Surgical Sciences \& Neurosciences, University of Siena, Siena, Italy.

Correspondence: D. Bennett, Respiratory Diseases Unit, Medical and Surgical Sciences \& Neurosciences, University of Siena, "Le Scotte" Hospital, Viale Bracci, 16, 53100, Siena, Italy. E-mail: david.btt@gmail.com

Received: June 192013 | Accepted after revision: Sept 192013 | First published online: Oct 102013

Conflict of interest: None declared.

\section{References}

Ibrahim WH. Helicobacter pylori eradication in the management of idiopathic pulmonary fibrosis. Eur Respir $J$ 2007; 30: 395-396.

2 Raghu G, Freudenberger TD, Yang S, et al. High prevalence of abnormal acid gastro-oesophageal reflux in idiopathic pulmonary fibrosis. Eur Respir J 2006; 27: 136-142.

3 Savarino E, Carbone R, Marabotto E, et al. Gastro-oesophageal reflux and gastric aspiration in idiopathic pulmonary fibrosis patients. Eur Respir J 2013; 42: 1322-1331.

4 Kanbay M, Kanbay A, Boyacioglu S. Helicobacter pylori infection as a possible risk factor for respiratory system disease: a review of the literature. Respir Med 2007; 101: 203-209.

5 Figura N, Gennari L, Merlotti D, et al. Prevalence of Helicobacter pylori infection in male patients with osteoporosis and controls. Dig Dis Sci 2005; 50: 847-852.

Bhandari A, Crowe SE. Helicobacter pylori in gastric malignancies. Curr Gastroenterol Rep 2012; 14: 489-496.

Suzuki H, Franceschi F, Nishizawa T, et al. Extragastric manifestations of Helicobacter pylori infection. Helicobacter 2011; 16: Suppl. 1, 65-69.

8 Gencer M, Ceylan E, Yildiz Zeyrek F, et al. Helicobacter pylori seroprevalence in patients with chronic obstructive pulmonary disease and its relation to pulmonary function tests. Respiration 2007; 74: 170-175.

9 Zhuo WL, Zhu B, Xiang ZL, et al. Assessment of the relationship between Helicobacter pylori and lung cancer: a meta-analysis. Arch Med Res 2009; 40: 406-410.

10 Fullerton D, Britton JR, Lewis SA, et al. Helicobacter pylori and lung function, asthma, atopy and allergic disease - a population-based cross-sectional study in adults. Int J Epidemiol 2009; 38: 419-426.

11 Raghu G, Collard HR, Egan JJ, et al. An official ATS/ERS/JRS/ALAT Statement: idiopathic pulmonary fibrosis: evidence-based guidelines for diagnosis and management. Am J Respir Crit Care Med 2011; 183: 788-824.

12 Gonnelli S, Caffarelli C, Tanzilli L, et al. The associations of body composition and fat distribution with bone mineral density in elderly Italian men and women. J Clin Densitom 2013; 16: 168-177.

13 Hogaboam, C. Pathogenesis of IPF. The 6th WASOG Conference, June 6th-7th 2013, Paris, France. www. wasog2013.com/Program Date last accessed: November 5, 2013.

\section{Combined pulmonary fibrosis and emphysema syndrome associated with ABCA3 mutations}

To the Editor:

Herein, we present the first report of combined pulmonary fibrosis and emphysema (CPFE) in an adult patient who was compound heterozygous for mutations of the ATP-binding cassette subfamily A member 3 gene (ABCA3, MIM 601615).

A 41-year-old nonsmoking male presented with dyspnoea on mild exertion. The patient's medical history indicated neonatal respiratory distress, gastro-oesophageal reflux and pneumonia 8 years previously that resolved with antibiotics. His physical examination revealed a mild pectus excavatum, finger clubbing and bilateral basal crackles. High-resolution computed tomography (HRCT) of the chest showed voluminous 
emphysema in the upper zones of the lungs associated with honeycomb fibrosis and ground-glass opacity in lower lobes, predominating in left lung (fig. 1). The bronchoalveolar lavage differential cell count was $67 \%$ macrophages, $22 \%$ neutrophils and $8 \%$ lymphocytes. Pulmonary function tests showed: total lung capacity of $75 \%$, vital capacity (VC) of $50 \%$, residual volume of $134 \%$; forced expiratory volume in $1 \mathrm{~s}(\mathrm{FEV} 1)$ of $49 \%$, diffusing capacity of the lung for carbon monoxide of $38 \%$ predicted, $\mathrm{FEV} 1 / \mathrm{VC}$ of $74 \%$, and arterial oxygen tension at room air was $96 \mathrm{mmHg}$. During a 6-min walk test the peripheral oxygen saturation decreased from $96 \%$ at rest to $90 \%$ after $630 \mathrm{~m}$ (80\% of predicted value). A lung biopsy was not performed. Doppler echocardiography showed normal heart cavities, with estimated systolic pulmonary arterial pressure of $37 \mathrm{mmHg}$. Serum $\alpha_{1}$-antitrypsin levels, autoimmune markers (including anti-nuclear antibody and rheumatoid factor), and immunoglobulin pattern were normal.

This clinical presentation of CPFE in a young patient prompted us to screen mutations in genes causing surfactant dysfunction. After informed consent was obtained, sequencing analysis of the surfactant protein C gene (SFTPC, MIM 178620) revealed no mutation. Sequence analysis of the ABCA3 gene identified two mutations: 1) c.3081_3092delinsCG resulting in a serine to valine change at codon 1028 with the creation of a stop codon 103 amino acids downstream (p.Ser1028Valfs ${ }^{\star} 103$ ); and 2) the common mutation c.875A $>$ T changing a glutamic acid to a valine at codon 292 (p.Glu292Val). None of these mutations were found in either the public polymorphism database or our controls. 2 years after presentation, chest HRCT as well as lung function worsened and azithromycin ( $250 \mathrm{mg}$ every other day) was initiated.

Pulmonary surfactant, a complex mixture of lipids and specific proteins located at the air-liquid interface, lowers alveolar surface tension thereby preventing alveolar collapse at the end of expiration. It is synthesised by alveolar type II cells, stored in lamellar bodies, and secreted by exocytosis. ABCA3 is expressed in the lamellar bodies of alveolar type II cells and is crucial to pulmonary surfactant storage and homeostasis. Several studies indicated a role of genes involved in surfactant metabolism in the development of diffuse lung diseases [1].

CPFE is a syndrome characterised by the coexistence of emphysema and pulmonary fibrosis in the same patient [2]. It typically occurs in male smokers and is associated with dyspnoea, upper lobe emphysema, lower lobe fibrosis and abnormalities of gas exchange. In the absence of the SFTPC mutation, previously associated with CPFE [3], we decided to analyse other genes involved in surfactant metabolism, such as $A B C A 3$. Recessive loss-of-function mutations in $A B C A 3$ present as lethal surfactant deficiency in the newborn, whereas other recessive mutations in $A B C A 3$ can result in interstitial lung disease in older children [4]. Previous studies showed that homozygous or compound heterozygous $A B C A 3$ mutations led
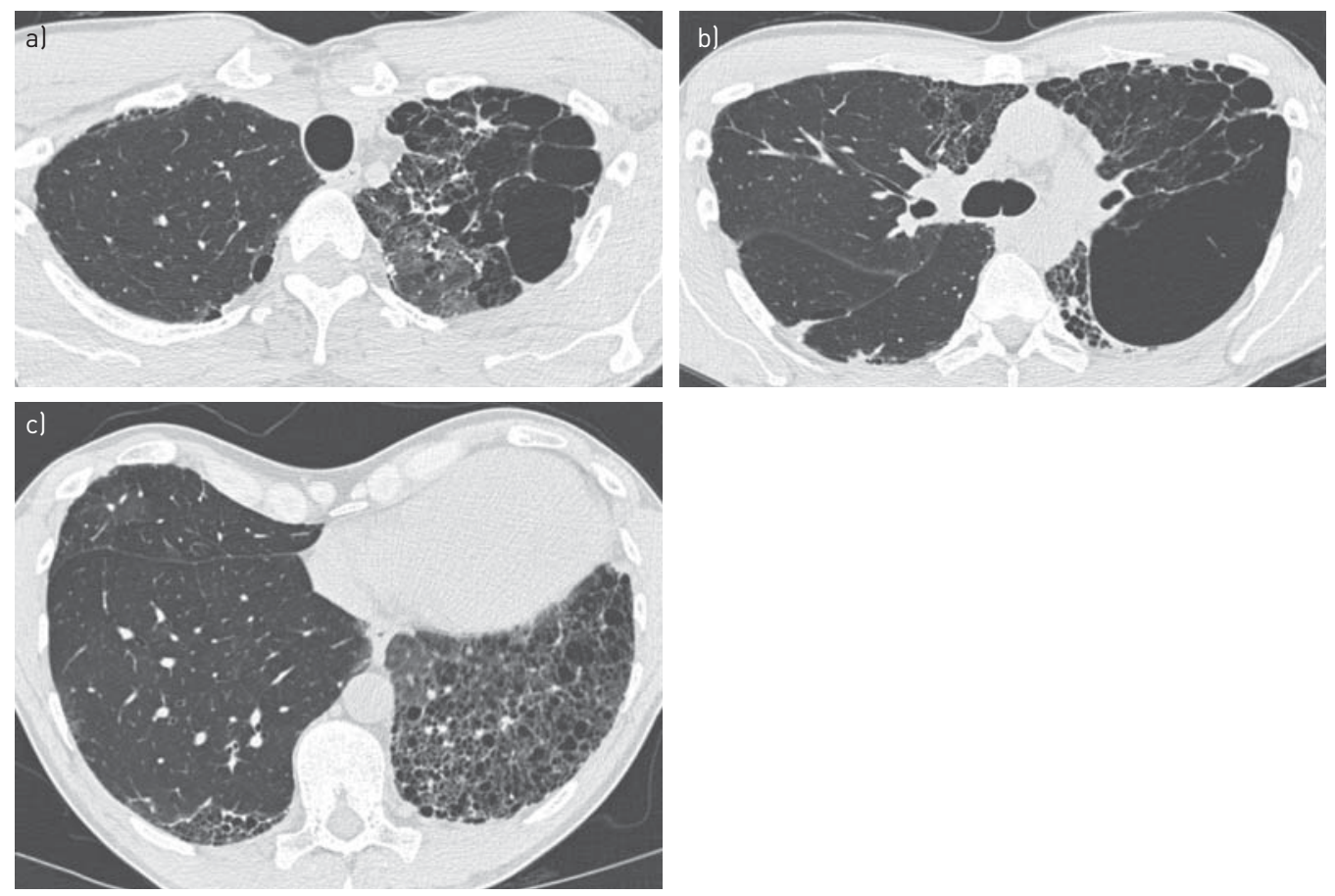

FIGURE 1 High-resolution computed tomography of the chest showing a, b) left upper lobe predominant emphysema associated with a, c) ground-glass opacities and c) asymmetric honeycomb pattern in the lower zones. 
to abnormal processing and/or trafficking of the ABCA3 protein [5], alterations in $\mathrm{ABCA} 3$ protein functions such as ATPase activity [6], or impaired lipid transport [7]. As previously described, our patient had a less severe phenotype than is usually associated with $A B C A 3$ mutations [4]. These variations in the clinical and radiological features may be related to the nature of the mutation. Our patient was found to be compound heterozygous for $A B C A 3$ mutations. The first is the common mutation p.Glu292Val, which is found in heterozygous form with a frequency of $<1 \%$ [8] and has been previously reported to be associated with mild lung disease. The second has not yet been described but is expected to be a disease-causing mutation as it introduces a premature termination codon, likely to be associated with markedly reduced mRNA levels due to nonsense-mediated degradation. Such a "null" allele precludes any functional ABCA3 from being made resulting in abnormal lamellar bodies, but should be less deleterious in combination with the mild mutation p.Glu292Val. Interactions with variants in other genes and/or with external factors such as viral or bacterial infections, as observed in our case, may also influence the observed phenotype [9].

The phenotype of our patient is very similar to that observed in the case reported by COTTIN et al. [3] carrying SFTPC mutations. Our patient is a nonsmoker and the emphysematous lesions were voluminous and localised, mostly in the upper lobes, whereas asymmetric fibrosis lesions were predominant in the lower lobes.

There is no specific treatment for CPFE syndrome. Supported immunosuppressive therapy was not indicated in this case without evidence of active inflammation. Improvement of severe interstitial lung disease in a young patient with ABCA3 deficiency has been reported after treatment with azithromycin, an azalide macrolide antibiotic characterised by a nitrogen in the macrolide ring [10]. Although, there is no evidence of efficacy of azithromycin in CPFE, the worsening of our patient's respiratory status together with the safety of this drug incited us to initiate this treatment in our patient.

To our knowledge, this is the first report of a phenotype of CPFE syndrome in an adult patient carrying mutations of the $A B C A 3$ gene. Although further studies are needed to confirm the role of surfactant metabolism in CPFE, this result suggests that this syndrome may have an underlying genetic predisposition.

0 @ERSpublications

The first report of combined pulmonary fibrosis and emphysema in an adult carrying compound heterozygote ABCA3 mutations http://ow.ly/rm0Dd

Ralph Epaud ${ }^{1,2,3}$, Céline Delestrain ${ }^{1}$, Malek Louha ${ }^{4}$, Stéphanie Simon ${ }^{1,2}$, Pascale Fanen ${ }^{1,2}$ and Abdellatif Tazi ${ }^{5,6}$

${ }^{1}$ Inserm, U955, Equipe 11, Créteil, ${ }^{2}$ Université Paris-Est, UMR_S955, UPEC, Créteil, ${ }^{3}$ Centre Hospitalier Intercommunal de Créteil, Service de Pédiatrie, Créteil, ${ }^{4}$ AP-HP, Hôpital Armand Trousseau, Service de Biochimie-Génétique Moléculaire, Paris, ${ }^{5}$ Université Paris Diderot, Paris, and ${ }^{6}$ INSERM UMR 717, AP-HP, Service de Pneumologie, Hôpital Saint Louis, Paris, France.

Correspondence: R. Epaud, Service de Pédiatrie, Centre Hospitalier Intercommunal, 40 avenue de Verdun, 94000 Créteil, France. E-mail: ralph.epaud@chicreteil.fr

Received: Aug 192013 | Accepted after revision: Sept 212013 | First published online: Oct 172013

Support statement: This work was supported by INSERM (P. Fanen, R. Epaud and C. Delestrain), Assistance PubliqueHôpitaux de Paris (A. Tazi) and the Société de Pneumologie de Langue Française (C. Delestrain). ABCA3 gene analysis technic was designed and initiated for Surfactant Disorders and Chronic Lung Disease (APSE; ClinicalTrials.gov identifier NCT00783978) (R. Epaud and M. Louha).

Conflict of interest: None declared.

\section{References}

Deutsch GH, Young LR, Deterding RR, et al. Diffuse lung disease in young children: application of a novel classification scheme. Am J Respir Crit Care Med 2007; 176: 1120-1128.

2 Cottin V, Nunes H, Brillet PY, et al. Combined pulmonary fibrosis and emphysema: a distinct under recognised entity. Eur Respir J 2005; 26: 586-593.

3 Cottin V, Reix P, Khouatra C, et al. Combined pulmonary fibrosis and emphysema syndrome associated with familial SFTPC mutation. Thorax 2011; 66: 918-919.

4 Flamein F, Riffault L, Muselet-Charlier C, et al. Molecular and cellular characteristics of ABCA3 mutations associated with diffuse parenchymal lung diseases in children. Hum Mol Genet 2011; 21: 765-775.

5 Cheong N, Madesh M, Gonzales LW, et al. Functional and trafficking defects in ATP binding cassette A3 mutants associated with respiratory distress syndrome. J Biol Chem 2006; 281: 9791-9800.

6 Matsumura Y, Ban N, Ueda K, et al. Characterization and classification of ATP-binding cassette transporter ABCA3 mutants in fatal surfactant deficiency. J Biol Chem 2006; 281: 34503-34514.

7 Matsumura Y, Ban N, Inagaki N. Aberrant catalytic cycle and impaired lipid transport into intracellular vesicles in ABCA3 mutants associated with nonfatal pediatric interstitial lung disease. Am J Physiol Lung Cell Mol Physiol 2008; 295: L698-L707. 
8 Wambach JA, Wegner DJ, Depass K, et al. Single ABCA3 mutations increase risk for neonatal respiratory distress syndrome. Pediatrics, 130: e1575-e1582.

9 Bullard JE, Nogee LM. Heterozygosity for ABCA3 mutations modifies the severity of lung disease associated with a surfactant protein C gene (SFTPC) mutation. Pediatr Res 2007; 62: 176-179.

10 Thouvenin G, Nathan N, Epaud R, et al. Diffuse parenchymal lung disease caused by surfactant deficiency: dramatic improvement by azithromycin. BMJ Case Rep 2013 [in press DOI: 10.1136/bcr-2013-009988].

\title{
Pulmonary arterial hypertension in familial hemiplegic migraine with ATP1A2 channelopathy
}

\begin{abstract}
To the Editor:
Pulmonary arterial hypertension (PAH) has been the focus of major research in recent years [1]. Involvement of mutations in genes encoding for members of the transforming growth factor- $\beta$ signalling pathway (BMPR2, ACVRL1, ENG and SMAD8) has been demonstrated in the development of heritable $\mathrm{PAH}$, allowing novel experimental and clinical approaches [2-4]. However, $\sim 30 \%$ of familial forms of PAH remain without any identification of genetic mutations. Recently, mutations of the KCNK3 gene (encoding $\mathrm{K}^{+}$channel subfamily $\mathrm{K}$ member 3 ) have been reported in patients with familial and sporadic PAH [5]. KCNK3 belongs to a family of mammalian $\mathrm{K}^{+}$channels, and are involved in the regulation of resting membrane potential, pulmonary vascular tone and in vascular remodelling. This result paves the way to the involvement of novel signalling pathways in the development of heritable PAH. Herein, we describe a novel association of PAH and a channelopathy due to mutation in ATP1A2 (encoding the $\alpha 2$-subunit of the $\mathrm{Na}^{+} / \mathrm{K}^{+}$-ATPase), a mutation known to cause familial hemiplegic migraine (FHM), a rare autosomal dominant disease [6].
\end{abstract}

A 24-year-old male was referred with a 1-year history of progressive exertional dyspnoea. Since the age of 8 years, he has reported recurrent episodes of hemiplegic migraine associated with muscle weakness and pain. The proband's mother (II4) (fig. 1) and two of his brothers (III6 and III7) had recurrent hemiplegic migraine with aura. There was no familial history of PAH. On admission, the patient was in New York Heart Association (NYHA) functional class III. His 6-min walk distance (6MWD) was 409 m. Pulmonary function tests were normal except for decreased diffusing capacity of the lungs for carbon monoxide. Doppler transthoracic echocardiography revealed signs of severe pulmonary hypertension with an estimated systolic pulmonary artery pressure of $75 \mathrm{mmHg}$, right ventricular dilatation and hypertrophy, and mild pericardial effusion. Right heart catheterisation confirmed pre-capillary pulmonary hypertension, with a mean pulmonary artery pressure $(\mathrm{mPAP})$ of $51 \mathrm{mmHg}$, a pulmonary capillary wedge pressure of $12 \mathrm{mmHg}$, a right atrial pressure of $7 \mathrm{mmHg}$, a cardiac index of $1.90 \mathrm{~L} \cdot \mathrm{min}^{-1} \cdot \mathrm{m}^{-2}$ and pulmonary vascular resistance (PVR) of 12.3 Wood units. No acute vasodilator response to nitric oxide was observed. Screening for other causes of pulmonary hypertension was negative. The patient was treated with a combination of intravenous epoprostenol, an endothelin receptor antagonist (ERA) and a phosphodiesterase type 5 inhibitor (PDE5i). The patient stopped taking the PDE5i after a few days because of side-effects, including increased symptoms of migraine. 4 months later, re-evaluation showed moderate clinical (NYHA functional class II and 6MWD $518 \mathrm{~m}$ ) and haemodynamic improvement (mPAP $43 \mathrm{mmHg}$, cardiac index $2.29 \mathrm{~L} \cdot \mathrm{min}^{-1} \cdot \mathrm{m}^{-2}$ and PVR 8.4 Wood units). The patient is still alive 1 year after diagnosis on intravenous epoprostenol and an ERA.

According to our local procedures, the patient underwent genetic counselling and gave written informed consent for genetic screening. No point mutations or large rearrangements of the BMPR2 and ACVRL1 genes were identified. To date, three genes (CACNA1A, ATP1A2 and SCNA1) encoding ion transporters are known to be associated with FHM. Genetic analysis revealed a nucleotide substitution in the coding sequence of the ATP1A2 gene (c.2819C>T; p.S940L) located on chromosome 1 (1q23). This mutation, which was not found in 200 control chromosomes, and was absent from the dbSNP, 1000 Genomes and Exome Sequencing Project data, affects a highly conserved amino acid, but has never been reported before. The patient's brothers, III4 and III6, were screened for the familial ATP1A2 mutation. Patient III4 did not carry the familial mutation and, as suggested by the clinical symptoms, the mutation was identified in patient III6 (fig. 1). Mutations of the ATP1A2 gene are known to cause FHM, a rare autosomal dominant disease characterised by migraine with motor weakness and aura [6]. Other neurological symptoms include 


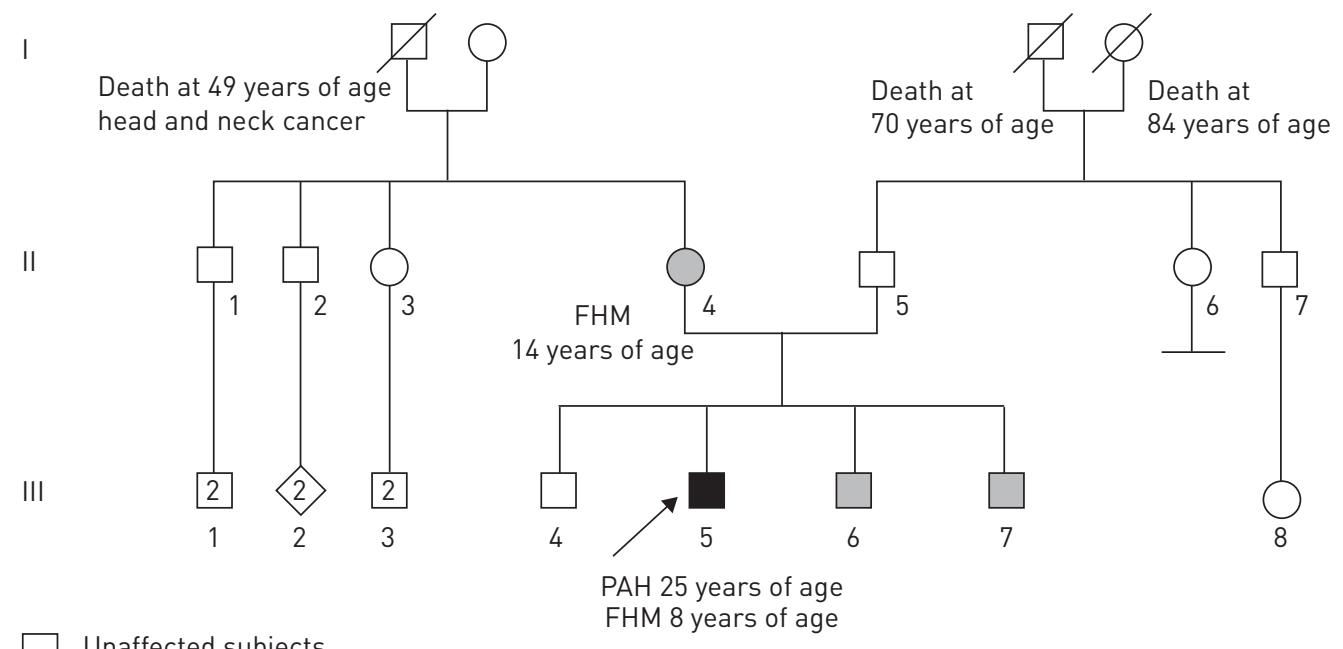

Unaffected subjects

$\mathrm{PAH}+\mathrm{FHM}$

FHM

FIGURE 1 Family tree. Ages indicated are those at pulmonary arterial hypertension (PAH) diagnosis or the beginning of familial hemiplegic migraine (FHM) symptoms. Arrow: proband.

various types of epileptic seizures and intellectual deficit; permanent cerebellar signs may be present in patients carrying a CACNA1A mutation [6]. FHM has an estimated prevalence of one in 20000, 20-30\% of cases carrying an ATP1A2 mutation [6]. In France, only 216 FHM patients (126 families) carry an ATP1A2 mutation (unpublished data).

The association of two rare diseases (PAH and FHM) supports the hypothesis of a potential common pathophysiological link. It is important to note that the presence and the activity of the $\alpha 2$-subunit of the $\mathrm{Na}^{+} / \mathrm{K}^{+}$-ATPase in lung and, more particularly, in pulmonary vascular smooth muscle cells have been previously reported [7]. In addition, several studies have reported substantial decreases in expression and/or activity of different types of $\mathrm{K}^{+}$channels in pulmonary arterial smooth muscle cells of patients displaying idiopathic $\mathrm{PAH}$, together with abnormalities in resting membrane potential and $\mathrm{Ca}^{2+}$ homeostasis [8]. Furthermore, pulmonary hypertension improvement has been demonstrated by restoring the expression of $\mathrm{K}^{+}$channels in a chronic hypoxic pulmonary hypertension rodent model (by treatment with a voltage-gated $\mathrm{K}^{+}(\mathrm{KV})$ channel activator or by gene transfer using adenovirus carrying the human Kv1.5 gene (KCNA5)) $[9,10]$. The chemical gradient produced by the normal activity of the $\mathrm{Na}^{+} / \mathrm{K}^{+}$-ATPase is important for restoration of low intracellular $\mathrm{Ca}^{2+}$ concentration. Inhibition of $\mathrm{K}^{+}$channels leads to an increase in intracellular $\mathrm{Ca}^{2+}$ concentration, which is a major stimulus for cell growth, migration and vasoconstriction [11]. Notably, inhibition of the $\mathrm{Na}^{+} / \mathrm{K}^{+}$-ATPase by ouabain rapidly activates the Ras/mitogen-activated protein kinase (MAPK) signalling pathway, leading to the proliferation of cultured vascular smooth cells [12, 13]. Interestingly, we have reported eight cases of neurofibromatosis type 1 and one case of Cowden syndrome associated with pre-capillary pulmonary hypertension [14]. Neurofibromatosis type 1 and Cowden syndrome are due to mutations in the NF1 and PTEN genes, respectively, leading to the activation of the Ras/MAPK signalling pathway and proliferation. Finally, it has been demonstrated that a decreased activity of $\mathrm{K}^{+}$channels can inhibit apoptosis by attenuating the activity of intracellular caspases [11]. Altogether, these observations support a possible role of mutations in ATP1A2 gene in the development of PAH through the disturbance of intracellular $\mathrm{Ca}^{2+}$ and $\mathrm{K}^{+}$concentrations.

We thus suggest that mutations in the ATP1A2 gene may contribute to pulmonary arterial remodelling and $\mathrm{PAH}$. However, we must emphasise that our report of a single family remains hypothesis-generating and requires future additional information. Importantly, no other families with mutations in ATP1A2 with a history of PAH have been reported to date. In addition, within the present family, the phenotype of FHM segregates with the ATP1A2 mutation, but only one member has PAH. While this family is intriguing, it remains possible that $\mathrm{PAH}$ is unrelated to the ATP1A2 mutation or FHM. Although these are both rare diseases, there is a small number of patients who do have coincidental rare diseases. In the future, our task will be to demonstrate the presence of ATP1A2 mutations in other individuals with PAH and/or functionally demonstrate how the mutation may affect the pulmonary vasculature. Similarly, patients displaying hereditary haemorrhagic telangiectasia and PAH have been very rarely reported in the past. 
Currently, $<50$ heritable PAH cases have been reported in ACVRL1 mutation carriers, while most mutation carriers develop hereditary haemorrhagic telangiectasia by the age of 60 years, emphasising that a single gene may cause different vascular diseases, alone or in combination, with markedly different penetrance [15]. In conclusion, our present case report reinforces the potential interest of ion channels in the pathogenesis of PAH.

@ERSpublications

This case report reinforces the potential interest of ion channels in the pathogenesis of PAH http://ow.ly/qkDD1

David Montani ${ }^{1,2,3}$, Barbara Girerd ${ }^{1,2,3}$, Sven Günther ${ }^{1,2,3}$, Florence Riant ${ }^{4,5}$, Elisabeth Tournier-Lasserve ${ }^{4,5}$, Laurent Magy ${ }^{6}$, Nizar Maazi ${ }^{7}$, Christophe Guignabert ${ }^{1,2,3}$, Laurent Savale ${ }^{1,2,3}$, Olivier Sitbon ${ }^{1,2,3}$, Gérald Simonneau ${ }^{1,2,3}$, Florent Soubrier ${ }^{8,9,10}$ and Marc Humbert ${ }^{1,2,3}$

${ }^{1}$ Université Paris-Sud, Le Kremlin-Bicêtre, ${ }^{2}$ Assistance Publique-Hôpitaux de Paris (AP-HP), Service de Pneumologie, DHU Thorax Innovation (DHU TORINO), Hôpital Bicêtre, Le Kremlin-Bicêtre, ${ }^{3}$ Institut National de la Santé et de la Recherche Médicale (INSERM) Unité Mixte de Recherche en Santé (UMR_S) 999, LabEx LERMIT, Centre Chirurgical Marie Lannelongue, Le Plessis Robinson, ${ }^{4}$ Service de Génétique Neuro-Vasculaire, AP-HP, Centre de Référence des Maladies Vasculaires Rares du Cerveau et de L'œil, Groupe Hospitalier Lariboisière - Fernand Widal, Paris, ${ }^{5}$ INSERM UMR_S 740, Université Paris Diderot, Sorbonne Paris Cité, Paris, ${ }^{6}$ Service de Neurologie, CHU de Limoges, Hôpital Dupuytren, Limoges, ${ }^{7}$ Service de Cardiologie, CHU de Limoges, Hôpital Dupuytren, Limoges, ${ }^{8}$ UMR_S 956, Université Pierre \& Marie Curie (UPMC), Université Paris 06, INSERM, Paris, ${ }^{9}$ Genetics Dept, Hôpital Pitié-Salpêtrière, AP-HP, Paris, and ${ }^{10}$ Institute for Cardiometabolism and Nutrition (ICAN), Paris, France.

Correspondence: D. Montani, Service de Pneumologie, Centre de Référence de l'Hypertension Pulmonaire Sévère, Hôpital de Bicêtre, AP-HP, 78 rue du Général Leclerc, 94275 Le Kremlin Bicêtre, France. E-mail: david.montani@ bct.aphp.fr

Received: Aug 222013 | Accepted after revision: Sept 242013 | First published online: Oct 172013

Conflict of interest: Disclosures can be found alongside the online version of this article at www.erj.ersjournals.com

Acknowledgements: The authors thank M. Eyries (Laboratoire d'Oncogénétique et Angiogénétique Moléculaire, Groupe Hospitalier Pitié-Salpétrière, Paris, France) for the genetic analysis of BMPR2 and ACVRL1.

\section{References}

1 Voelkel NF, Gomez-Arroyo J, Abbate A, et al. Pathobiology of pulmonary arterial hypertension and right ventricular failure. Eur Respir J 2012; 40: 1555-1565.

2 Liu D, Liu QQ, Eyries M, et al. Molecular genetics and clinical features of Chinese idiopathic and heritable pulmonary arterial hypertension patients. Eur Respir J 2012; 39: 597-603.

3 Frydman N, Steffann J, Girerd B, et al. Pre-implantation genetic diagnosis in pulmonary arterial hypertension due to BMPR2 mutation. Eur Respir J 2012; 39: 1534-1535.

4 Reynolds AM, Holmes MD, Danilov SM, et al. Targeted gene delivery of BMPR2 attenuates pulmonary hypertension. Eur Respir J 2012; 39: 329-343.

5 Ma L, Roman-Campos D, Austin ED, et al. A novel channelopathy in pulmonary arterial hypertension. $N$ Engl J Med 2013; 369: 351-361.

6 Russell MB, Ducros A. Sporadic and familial hemiplegic migraine: pathophysiological mechanisms, clinical characteristics, diagnosis, and management. Lancet Neurol 2011; 10: 457-470.

7 Ghosh B, Kar P, Mandal A, et al. $\mathrm{Ca}^{2+}$ influx mechanisms in caveolae vesicles of pulmonary smooth muscle plasma membrane under inhibition of $\alpha 2 \beta 1$ isozyme of $\mathrm{Na}^{+} / \mathrm{K}^{+}$-ATPase by ouabain. Life Sci 2009; 84: 139-148.

8 Yuan JX, Aldinger AM, Juhaszova M, et al. Dysfunctional voltage-gated $\mathrm{K}^{+}$channels in pulmonary artery smooth muscle cells of patients with primary pulmonary hypertension. Circulation 1998; 98: 1400-1406.

9 Morecroft I, Murray A, Nilsen M, et al. Treatment with the Kv7 potassium channel activator flupirtine is beneficial in two independent mouse models of pulmonary hypertension. Br J Pharmacol 2009; 157: 1241-1249.

10 Pozeg ZI, Michelakis ED, McMurtry MS, et al. In vivo gene transfer of the $\mathrm{O}_{2}$-sensitive potassium channel $\mathrm{Kv}_{\mathrm{v}} 1.5$ reduces pulmonary hypertension and restores hypoxic pulmonary vasoconstriction in chronically hypoxic rats. Circulation 2003; 107: 2037-2044.

11 Kuhr FK, Smith KA, Song MY, et al. New mechanisms of pulmonary arterial hypertension: role of $\mathrm{Ca}^{2+}$ signaling. Am J Physiol Heart Circ Physiol 2012; 302: H1546-H1562.

12 Aydemir-Koksoy A, Abramowitz J, Allen JC. Ouabain-induced signaling and vascular smooth muscle cell proliferation. J Biol Chem 2001; 276: 46605-46611.

13 Kometiani P, Li J, Gnudi L, et al. Multiple signal transduction pathways link $\mathrm{Na}^{+} / \mathrm{K}^{+}$-ATPase to growth-related genes in cardiac myocytes. The roles of Ras and mitogen-activated protein kinases. J Biol Chem 1998; 273: 15249-15256.

14 Montani D, Coulet F, Girerd B, et al. Pulmonary hypertension in patients with neurofibromatosis type I. Medicine (Baltimore) 2011; 90: 201-211.

15 Girerd B, Montani D, Coulet F, et al. Clinical outcomes of pulmonary arterial hypertension in patients carrying an ACVRL1 (ALK1) mutation. Am J Respir Crit Care Med 2010; 181: 851-861. 


\title{
Enhanced detection of Legionnaires' disease by PCR testing of induced sputum and throat swabs
}

\author{
To the Editor:
}

Legionnaires' disease, particularly that caused by non-pneumophila species, is notoriously underdiagnosed $[1,2]$. We recently found a four-fold increase in case detection of Legionnaires' disease through a laboratory-initiated strategy of systematic PCR testing for Legionella species of all lower respiratory specimens from patients with pneumonia or immune compromised status [3]. This strategy relies on the availability of lower respiratory specimens and the recording of relevant clinical information on laboratory requisition forms by clinicians. We recognised that this strategy will miss testing patients who could not expectorate sputum and when inadequate clinical information is written on laboratory requisition forms. To address this diagnostic gap we enhanced case detection by actively identifying patients with communityacquired pneumonia (CAP) and by collecting induced sputum from those unable to expectorate voluntarily. In addition, we evaluated throat swabs as an alternative specimen for PCR testing.

From October 2012 to March 2013 patients admitted to Christchurch Hospital and The Princess Margaret Hospital (both in Christchurch, New Zealand) with CAP and aged $\geqslant 18$ years were recruited. For logistical reasons, recruitment occurred on weekdays only. The study period was chosen to coincide with peak Legionnaires' disease activity in Christchurch [3]. Patients were excluded if the pneumonia was hospital acquired or associated with bronchial obstruction, bronchiectasis or tuberculosis. Patients were not eligible for sputum induction if they required high-flow oxygen or assisted ventilation at enrolment.

Ethical approval was obtained from the New Zealand Northern A Ethics Committee. Informed consent was obtained from the patient or their next of kin with separate informed consent for induced sputum collection.

Legionnaires' disease was defined by a positive sputum PCR, sputum culture or urinary antigen test result in a patient with pneumonia. CAP was defined as an acute illness with clinical features of pneumonia and radiographic pulmonary shadowing that is at least segmental or present in one lobe and is neither preexisting nor because of some other known cause.

Sputum induction was performed on the day it was requested, using ultrasonically nebulised 7\% hypertonic saline for 20 min via a DeVilbiss Ultraneb (DeVilbiss Healthcare, Somerset, PA, USA). Throat swabs were collected using nylon flocked swabs (Floqswabs; Copan flock technologies, Brescia, Italy) and placed in universal transport media. Sputum and throat swabs were tested for Legionella species using PCR. The isolation of DNA from clinical specimens was performed using the SPRI-TE (Beckman Coulter, Auckland, New Zealand) nucleic acid extractor and genomic DNA Extraction Kit as recommended by the manufacturer. Legionella DNA was detected using real-time PCR [2], confirmation of the PCR products that did not have melting curve data consistent with Legionella pneumophila was performed using a realtime PCR with a different gene target [3]. This confirmatory PCR had both genus and Legionella longbeachae specific probes. PCR inhibitor controls were used to validate negative PCR results [3-5]. Urine samples were tested for L. pneumophila serogroup 1 and Streptococcus pneumoniae using the BinaxNOW Legionella Urinary Antigen Card and BinaxNOW S. pneumoniae Antigen Card (Alere, Scarborough, ME, USA), respectively. Blood cultures were taken at the request of attending physicians.

Over the study period, 145 patients were eligible with 114 enrolled. 26 patients did not consent and four who were initially enrolled were excluded when the diagnosis of malignancy $(n=1)$, tuberculosis $(n=2)$ and hospital-acquired pneumonia $(n=1)$ was made. One patient was excluded because pneumonia was not the primary reason for admission.

The median (interquartile range) age of those included was 69 (18-101) years, with 67 (59\%) females. There were $22(19 \%)$ cases of Legionnaires' disease: 16 cases caused by L. longbeachae, three caused by L. pneumophila and three non-typable Legionella species. Of these, 21 were detected by sputum PCR, eight had positive sputum cultures (all PCR positive), and three had positive urinary antigen tests (two were also detected with sputum). The three non-typable Legionella species were all culture negative, urinary antigen 


$\begin{array}{lc}\text { Expectorated sputum } & 13 / 68 \\ \text { Expectorated sputum obtained with nurse or } & 4 / 10 \\ \quad \text { physiotherapist assistance } & \\ \text { Induced sputum } & 4 / 19 \\ \text { Throat swab } & 3 / 114\end{array}$

Data are presented as $\mathrm{n} / \mathrm{N}$.

negative and of insufficient copy number to type. Of the 114 patients, $17(15 \%)$ had positive urinary antigen tests for S. pneumoniae and one (1\%) patient had a positive blood culture for S. pneumoniae.

A summary of the yield of diagnostic specimens is presented in table 1 . Of the 114 cases, 46 (40\%) were unable to spontaneously expectorate sputum. Induced sputum was requested for 31 patients and 15 did not give consent or were ineligible. Of the 31 who consented to have sputum induced, $10(34 \%)$ produced an expectorated sputum sample with nursing or physiotherapist assistance (four were positive for Legionella), 19 underwent sputum induction and two were discharged prior to the procedure. Of the 19 who underwent sputum induction, all produced sputum without adverse events and four tested positive for Legionella. Therefore, eight (36\%) out of 22 cases of Legionnaires' disease would not have been detected without active specimen collection. Of the PCR-positive sputum specimens, $11(52 \%)$ out of 21 had $>10$ squamous epithelial cells per low power field and $12(57 \%)$ out of 21 had $<25$ leukocytes per low power field; criteria often used to indicate poor quality specimens.

Throat swabs were performed on all 114 patients and were positive in only three (3\%), all of whom also had positive sputum specimens.

The identification of Legionella spp. altered the treatment in $16(73 \%)$ patients. In five patients this was due to the inclusion of an agent active against Legionella and in 11 patients there was a rationalisation of antimicrobials.

We found that active specimen collection increased the case detection of Legionnaires' disease in our region by at least one-third over and above PCR testing of routine specimens. During spring and summer in our region, Legionnaires' disease accounted for approximately one-fifth of all cases of adult CAP admitted to hospital, and was more common than confirmed pneumococcal pneumonia.

The diagnostic performance of Legionella PCR has been reported previously but, in brief, it is highly sensitive and specific, with a rapid turn-around time and disease severity correlates with bacterial load $[3,6]$.

Sputum is the preferred diagnostic sample for Legionella PCR testing [2]. However, $>40 \%$ of pneumonia patients in our study were unable to spontaneously expectorate sputum. We managed to obtain a sputum sample through extra assistance or induction in only approximately two-thirds of these cases but, in doing so, still increased the case detection of Legionnaires' disease by $36 \%$. The most common reason for not obtaining sputum was ineligibility for sputum induction. We were cautious in selecting patients for sputum induction to avoid adverse reactions and it was universally well-tolerated. The technique of induced sputum has been shown to be safe by others and could be more widely applied [7].

It has been reported that throat swabs may be useful for diagnosing the cause of lower respiratory tract infections by PCR [8-10]. In our study, a throat swab was positive in a minority of cases and offered no advantage over sputum samples.

The relatively small sample size and study period over only one spring/summer are important limitations of our study. Additionally, the conclusions are only valid in regions with a high incidence of non-pneumophila Legionnaires' disease such as Australasia.

In conclusion, active collection of sputum samples for PCR testing, including by induction, in patients with CAP enhances the case detection of Legionnaires' disease, and is recommended in high-prevalence regions. Throat swabs offer no advantage over sputum samples, but further work is needed to assess their potential role in situations when lower respiratory samples cannot be obtained.

@ERSpublications

Testing induced sputum samples from those who cannot produce sputum increases diagnosis of Legionnaires' disease http://ow.ly/rm9WD 
Michael J. Maze ${ }^{1,2}$, Sandy Slow ${ }^{3}$, Anne-Marie Cumins ${ }^{4}$, Kenjin Boon ${ }^{4}$, Patricia Goulter ${ }^{5}$, Roslyn G. Podmore ${ }^{6}$, Trevor P. Anderson ${ }^{6}$, Kevin Barratt ${ }^{6}$, Sheryl A. Young ${ }^{6}$, Alan D. Pithie ${ }^{1,4}$, Michael J. Epton ${ }^{2,7}$, Anja M. Werno ${ }^{6}$, Stephen T. Chambers ${ }^{1,3}$ and David R. Murdoch ${ }^{3,6}$

${ }^{1}$ Dept of Infectious Diseases, Christchurch Hospital, Christchurch, ${ }^{2}$ Dept of Respiratory Medicine, Christchurch Hospital, Christchurch, ${ }^{3}$ Dept of Pathology, University of Otago, Christchurch, ${ }^{4}$ Dept of General Medicine, Christchurch Hospital, Christchurch, ${ }^{5}$ Physiotherapy Dept, Christchurch Hospital, Christchurch, ${ }^{6}$ Microbiology Unit, Canterbury Health Laboratories, Christchurch, and ${ }^{7}$ Canterbury Respiratory Research Group, Christchurch, New Zealand.

Correspondence: M.J. Maze, Dept of Respiratory Medicine, Private Bag 4710, Christchurch, New Zealand. E-mail: Michael.Maze@cdhb.health.nz

Received: Nov 042013 | Accepted: Nov 142013 | First published online: Dec 52013

Conflict of interest: None declared.

Acknowledgements: We thank the staff of the General Medicine Service and the Physiotherapy Department of Christchurch Hospital, and of the Microbiology Unit of Canterbury Health Laboratories (both Christchurch, New Zealand) for their invaluable support throughout the project.

\section{References}

Diederen BM. Legionella spp. and Legionnaires' disease. J Infect 2008; 56: 1-12.

Murdoch DR. Diagnosis of Legionella infection. Clin Infect Dis 2003; 36: 64-69.

Murdoch DR, Podmore RG, Anderson TP, et al. Impact of routine systematic PCR testing on case finding for Legionnaires' disease: a pre-post comparison study. Clin Infect 2013; 57: 1275-1281.

4 Reischl U, Linde H-J, Lehn N, et al. Direct detection and differentiation of Legionella spp. and Legionella pneumophila in clinical specimens by dual-colour real-time PCR and melting curve analysis. J Clin Microbiol 2002; 40: 3814-3817.

5 Yang G, Benson R, Pelish T, et al. Dual detection of Legionella pneumophila and Legionella species by real-time PCR targeting the 23S-5S rRNA gene spacer region. Clin Microbiol Infect 2010; 16: 255-261.

6 Diederen BM, Kluytmans AJW, Vandenbroucke-Grauls CM, et al. Utility of real-time PCR for diagnosis of Legionnaires' disease in routine clinical practice. J Clin Microbiol 2008; 46: 671-677.

$7 \quad$ Pizzichini E, Pizzichini MMM, Leigh R, et al. Safety of sputum induction. Eur Respir J 2002; 20: Suppl. 37, 9s-18s.

8 Diederen BM, Peeters MF. Are oropharyngeal swabs suitable as samples for Legionella-specific PCR testing? J Clin Microbiol 2007; 45: 3482-3483.

9 McDonough EA, Metzgar D, Hansen CJ, et al. A cluster of Legionella-associated pneumonia cases in a population of military recruits. J Clin Microbiol 2007; 45: 2075-2077.

10 Ramirez JA, Ahkee S, Tolentino A, et al. Diagnosis of Legionella pneumophila, Mycoplasma pneumoniae, or Chlamydia pneumoniae lower respiratory infection using the polymerase chain reaction on a single throat swab specimen. Diagn Microbiol Infect Dis 1996; 24: 7-14. 